\title{
Formalization of the Data Encryption Standard $^{1}$
}

\author{
Hiroyuki Okazaki \\ Shinshu University \\ Nagano, Japan
}

\author{
Yasunari Shidama \\ Shinshu University \\ Nagano, Japan
}

Summary. In this article we formalize DES (the Data Encryption Standard), that was the most widely used symmetric cryptosystem in the world. DES is a block cipher which was selected by the National Bureau of Standards as an official Federal Information Processing Standard for the United States in 1976 [15].

MML identifier: DESCIP_1, version: $\underline{7.12 .02 \quad 4.181 .1147}$

The papers [14], [5], [12], [1], [16], [4], [6], [18], [11], [7], [8], [17], [20], [2], [3], $[9],[21],[22],[13],[19]$, and [10] provide the terminology and notation for this paper.

\section{Preliminaries}

Let $n$ be a natural number and let $f$ be an $n$-element finite sequence. Note that $\operatorname{Rev}(f)$ is $n$-element.

Let $D$ be a non empty set, let $n$ be a natural number, and let $f$ be an element of $D^{n}$. Then $\operatorname{Rev}(f)$ is an element of $D^{n}$.

Let $n$ be a natural number and let $f$ be a finite sequence. We introduce Op-Left $(f, n)$ as a synonym of $f\lceil n$. We introduce Op-Right $(f, n)$ as a synonym of $f_{l n}$.

Let $D$ be a non empty set, let $n$ be a natural number, and let $f$ be a finite sequence of elements of $D$. Then $\operatorname{Op}-\operatorname{Left}(f, n)$ is a finite sequence of elements of $D$. Then Op-Right $(f, n)$ is a finite sequence of elements of $D$.

\footnotetext{
${ }^{1}$ This work was supported by JSPS KAKENHI 21240001.
}

(C) 2012 University of Białystok CC-BY-SA License ver. 3.0 or later ISSN $1426-2630(\mathrm{p}), 1898-9934(\mathrm{e})$ 
Let $D$ be a non empty set, let $n$ be a natural number, and let $s$ be an element of $D^{2 \cdot n}$. We introduce SP-Left $s$ as a synonym of Op-Left $(s, n)$. We introduce SP-Right $s$ as a synonym of Op-Right $(s, n)$.

Let $D$ be a non empty set, let $n$ be a natural number, and let $s$ be an element of $D^{2 \cdot n}$. Then SP-Left $s$ is an element of $D^{n}$.

One can prove the following propositions:

(1) For all non empty elements $m, n$ of $\mathbb{N}$ and for every element $s$ of $D^{n}$ such that $m \leq n$ holds Op-Left $(s, m)$ is an element of $D^{m}$.

(2) Let $m, n, l$ be non empty elements of $\mathbb{N}$ and $s$ be an element of $D^{n}$. If $m \leq n$ and $l=n-m$, then Op-Right $(s, m)$ is an element of $D^{l}$.

Let $D$ be a non empty set, let $n$ be a non empty element of $\mathbb{N}$, and let $s$ be an element of $D^{2 \cdot n}$. Then SP-Right $s$ is an element of $D^{n}$.

Next we state the proposition

(3) For every non empty element $n$ of $\mathbb{N}$ and for every element $s$ of $D^{2 \cdot n}$ holds (SP-Left $s) \frown$ SP-Right $s=s$.

Let $s$ be a finite sequence. The functor Op-LeftShift $s$ yielding a finite sequence is defined by:

(Def. 1) Op-LeftShift $s=\left(s_{\lfloor 1}\right)^{\frown}\langle s(1)\rangle$.

Next we state three propositions:

(4) For every finite sequence $s$ such that $1 \leq$ len $s$ holds len Op-LeftShift $s=$ len $s$.

(5) If $1 \leq$ len $s$, then Op-LeftShift $s$ is a finite sequence of elements of $D$ and len Op-LeftShift $s=$ len $s$.

(6) For every non empty element $n$ of $\mathbb{N}$ and for every element $s$ of $D^{n}$ holds Op-LeftShift $s$ is an element of $D^{n}$.

Let $s$ be a finite sequence. The functor Op-RightShift $s$ yields a finite sequence and is defined by:

(Def. 2) Op-RightShift $s=(\langle s($ len $s)\rangle \frown s) \uparrow$ len $s$.

One can prove the following three propositions:

(7) For every finite sequence $s$ holds len Op-RightShift $s=$ len $s$.

(8) If $1 \leq$ len $s$, then Op-RightShift $s$ is a finite sequence of elements of $D$ and len Op-RightShift $s=$ len $s$.

(9) For every non empty element $n$ of $\mathbb{N}$ and for every element $s$ of $D^{n}$ holds Op-RightShift $s$ is an element of $D^{n}$.

Let $D$ be a non empty set, let $s$ be a finite sequence of elements of $D$, and let $n$ be an integer. Let us assume that $1 \leq$ len $s$. The functor Op-Shift $(s, n)$ yields a finite sequence of elements of $D$ and is defined by:

(Def. 3) len Op-Shift $(s, n)=\operatorname{len} s$ and for every natural number $i$ such that $i \in$ Seg len $s$ holds $(\mathrm{Op}-\operatorname{Shift}(s, n))(i)=s(((i-1)+n) \bmod$ len $s)+1)$. 
The following propositions are true:

(10) For all integers $n, m$ such that $1 \leq$ len $s$ holds Op-Shift(Op-Shift $(s, n), m)=$ Op-Shift $(s, n+m)$.

(11) If $1 \leq$ len $s$, then Op-Shift $(s, 0)=s$.

(12) If $1 \leq$ len $s$, then Op-Shift $(s$, len $s)=s$.

(13) If $1 \leq$ len $s$, then Op-Shift $(s,-$ len $s)=s$.

(14) Let $n$ be a non empty element of $\mathbb{N}, m$ be an integer, and $s$ be an element of $D^{n}$. Then Op-Shift $(s, m)$ is an element of $D^{n}$.

(15) If $1 \leq$ len $s$, then Op-Shift $(s,-1)=$ Op-RightShift $s$.

(16) If $1 \leq$ len $s$, then Op-Shift $(s, 1)=$ Op-LeftShift $s$.

Let $x, y$ be elements of Boolean ${ }^{28}$. Then $x^{\frown} y$ is an element of Boolean ${ }^{56}$.

Let $n$ be a non empty element of $\mathbb{N}$, let $s$ be an element of Boolean ${ }^{n}$, and let $i$ be a natural number. Then $s(i)$ is an element of Boolean.

Let $n$ be a non empty element of $\mathbb{N}$, let $s$ be an element of $\mathbb{N}^{n}$, and let $i$ be a natural number. Then $s(i)$ is an element of $\mathbb{N}$.

Let $n$ be a natural number. Observe that every element of Boolean ${ }^{n}$ is boolean-valued.

Let $n$ be an element of $\mathbb{N}$ and let $s, t$ be elements of Boolean ${ }^{n}$. We introduce $\mathrm{Op}-\mathrm{XOR}(s, t)$ as a synonym of $s \oplus t$.

Let $n$ be a non empty element of $\mathbb{N}$ and let $s, t$ be elements of Boolean ${ }^{n}$. Then $\operatorname{Op}-\operatorname{XOR}(s, t)$ is an element of Boolean ${ }^{n}$ and it can be characterized by the condition:

(Def. 4) For every natural number $i$ such that $i \in \operatorname{Seg} n$ holds $(\mathrm{Op}-\mathrm{XOR}(s, t))(i)=s(i) \oplus t(i)$.

Let us notice that the functor $\operatorname{Op}-\operatorname{XOR}(s, t)$ is commutative.

Let $n, k$ be non empty elements of $\mathbb{N}$, let $R_{1}$ be an element of $\left(\text { Boolean }^{n}\right)^{k}$, and let $i$ be an element of Seg $k$. Then $R_{1}(i)$ is an element of Boolean ${ }^{n}$.

We now state the proposition

(17) For every non empty element $n$ of $\mathbb{N}$ and for all elements $s, t$ of Boolean ${ }^{n}$ holds Op-XOR $(\mathrm{Op}-\mathrm{XOR}(s, t), t)=s$.

Let $m$ be a non empty element of $\mathbb{N}$, let $D$ be a non empty set, let $L$ be a sequence of $D^{m}$, and let $i$ be a natural number. Then $L(i)$ is an element of $D^{m}$.

Let $f$ be a function from 64 into 16 and let $i$ be a set. Then $f(i)$ is an element of 16 .

Next we state the proposition

(18) For all natural numbers $n, m$ such that $n+m \leq$ len $s$ holds $\left(s\lceil n)^{\frown}\right.$ $\left(s_{\lfloor n}\lceil m)=s\lceil(n+m)\right.$.

The scheme QuadChoiceRec deals with non empty sets $\mathcal{A}, \mathcal{B}, \mathcal{C}, \mathcal{D}$, an element $\mathcal{E}$ of $\mathcal{A}$, an element $\mathcal{F}$ of $\mathcal{B}$, an element $\mathcal{G}$ of $\mathcal{C}$, an element $\mathcal{H}$ of $\mathcal{D}$, and a 9-ary predicate $\mathcal{P}$, and states that: 
There exists a function $f$ from $\mathbb{N}$ into $\mathcal{A}$ and there exists a function $g$ from $\mathbb{N}$ into $\mathcal{B}$ and there exists a function $h$ from $\mathbb{N}$ into $\mathcal{C}$ and there exists a function $i$ from $\mathbb{N}$ into $\mathcal{D}$ such that $f(0)=\mathcal{E}$ and $g(0)=\mathcal{F}$ and $h(0)=\mathcal{G}$ and $i(0)=\mathcal{H}$ and for every element $n$ of $\mathbb{N}$ holds $\mathcal{P}[n, f(n), g(n), h(n), i(n), f(n+1), g(n+1), h(n+1), i(n+1)]$ provided the following condition is satisfied:

- Let $n$ be an element of $\mathbb{N}, x$ be an element of $\mathcal{A}, y$ be an element of $\mathcal{B}, z$ be an element of $\mathcal{C}$, and $w$ be an element of $\mathcal{D}$. Then there exists an element $x_{1}$ of $\mathcal{A}$ and there exists an element $y_{1}$ of $\mathcal{B}$ and there exists an element $z_{1}$ of $\mathcal{C}$ and there exists an element $w_{1}$ of $\mathcal{D}$ such that $\mathcal{P}\left[n, x, y, z, w, x_{1}, y_{1}, z_{1}, w_{1}\right]$.

Next we state a number of propositions:

(19) Let $x$ be a set. Suppose $x \in \operatorname{Seg} 16$. Then $x=1$ or $x=2$ or $x=3$ or $x=4$ or $x=5$ or $x=6$ or $x=7$ or $x=8$ or $x=9$ or $x=10$ or $x=11$ or $x=12$ or $x=13$ or $x=14$ or $x=15$ or $x=16$.

(20) Let $x$ be a set. Suppose $x \in \operatorname{Seg} 32$. Then $x=1$ or $x=2$ or $x=3$ or $x=4$ or $x=5$ or $x=6$ or $x=7$ or $x=8$ or $x=9$ or $x=10$ or $x=11$ or $x=12$ or $x=13$ or $x=14$ or $x=15$ or $x=16$ or $x=17$ or $x=18$ or $x=19$ or $x=20$ or $x=21$ or $x=22$ or $x=23$ or $x=24$ or $x=25$ or $x=26$ or $x=27$ or $x=28$ or $x=29$ or $x=30$ or $x=31$ or $x=32$.

(21) Let $x$ be a set. Suppose $x \in \operatorname{Seg} 48$. Then $x=1$ or $x=2$ or $x=3$ or $x=4$ or $x=5$ or $x=6$ or $x=7$ or $x=8$ or $x=9$ or $x=10$ or $x=11$ or $x=12$ or $x=13$ or $x=14$ or $x=15$ or $x=16$ or $x=17$ or $x=18$ or $x=19$ or $x=20$ or $x=21$ or $x=22$ or $x=23$ or $x=24$ or $x=25$ or $x=26$ or $x=27$ or $x=28$ or $x=29$ or $x=30$ or $x=31$ or $x=32$ or $x=33$ or $x=34$ or $x=35$ or $x=36$ or $x=37$ or $x=38$ or $x=39$ or $x=40$ or $x=41$ or $x=42$ or $x=43$ or $x=44$ or $x=45$ or $x=46$ or $x=47$ or $x=48$.

(22) Let $x$ be a set. Suppose $x \in \operatorname{Seg} 56$. Then $x=1$ or $x=2$ or $x=3$ or $x=4$ or $x=5$ or $x=6$ or $x=7$ or $x=8$ or $x=9$ or $x=10$ or $x=11$ or $x=12$ or $x=13$ or $x=14$ or $x=15$ or $x=16$ or $x=17$ or $x=18$ or $x=19$ or $x=20$ or $x=21$ or $x=22$ or $x=23$ or $x=24$ or $x=25$ or $x=26$ or $x=27$ or $x=28$ or $x=29$ or $x=30$ or $x=31$ or $x=32$ or $x=33$ or $x=34$ or $x=35$ or $x=36$ or $x=37$ or $x=38$ or $x=39$ or $x=40$ or $x=41$ or $x=42$ or $x=43$ or $x=44$ or $x=45$ or $x=46$ or $x=47$ or $x=48$ or $x=49$ or $x=50$ or $x=51$ or $x=52$ or $x=53$ or $x=54$ or $x=55$ or $x=56$.

(23) Let $x$ be a set. Suppose $x \in \operatorname{Seg} 64$. Then $x=1$ or $x=2$ or $x=3$ or $x=4$ or $x=5$ or $x=6$ or $x=7$ or $x=8$ or $x=9$ or $x=10$ or $x=11$ or $x=12$ or $x=13$ or $x=14$ or $x=15$ or $x=16$ or $x=17$ or $x=18$ or $x=19$ or $x=20$ or $x=21$ or $x=22$ or $x=23$ or $x=24$ or $x=25$ or 
$x=26$ or $x=27$ or $x=28$ or $x=29$ or $x=30$ or $x=31$ or $x=32$ or $x=33$ or $x=34$ or $x=35$ or $x=36$ or $x=37$ or $x=38$ or $x=39$ or $x=40$ or $x=41$ or $x=42$ or $x=43$ or $x=44$ or $x=45$ or $x=46$ or $x=47$ or $x=48$ or $x=49$ or $x=50$ or $x=51$ or $x=52$ or $x=53$ or $x=54$ or $x=55$ or $x=56$ or $x=57$ or $x=58$ or $x=59$ or $x=60$ or $x=61$ or $x=62$ or $x=63$ or $x=64$.

(24) For every non empty natural number $n$ holds $n=\{0\} \cup(\operatorname{Seg} n \backslash\{n\})$.

(25) For every non empty natural number $n$ and for every set $x$ such that $x \in n$ holds $x=0$ or $x \in \operatorname{Seg} n$ and $x \neq n$.

(26) Let $x$ be a set. Suppose $x \in 16$. Then $x=0$ or $x=1$ or $x=2$ or $x=3$ or $x=4$ or $x=5$ or $x=6$ or $x=7$ or $x=8$ or $x=9$ or $x=10$ or $x=11$ or $x=12$ or $x=13$ or $x=14$ or $x=15$.

(27) Let $x$ be a set. Suppose $x \in 64$. Then $x=0$ or $x=1$ or $x=2$ or $x=3$ or $x=4$ or $x=5$ or $x=6$ or $x=7$ or $x=8$ or $x=9$ or $x=10$ or $x=11$ or $x=12$ or $x=13$ or $x=14$ or $x=15$ or $x=16$ or $x=17$ or $x=18$ or $x=19$ or $x=20$ or $x=21$ or $x=22$ or $x=23$ or $x=24$ or $x=25$ or $x=26$ or $x=27$ or $x=28$ or $x=29$ or $x=30$ or $x=31$ or $x=32$ or $x=33$ or $x=34$ or $x=35$ or $x=36$ or $x=37$ or $x=38$ or $x=39$ or $x=40$ or $x=41$ or $x=42$ or $x=43$ or $x=44$ or $x=45$ or $x=46$ or $x=47$ or $x=48$ or $x=49$ or $x=50$ or $x=51$ or $x=52$ or $x=53$ or $x=54$ or $x=55$ or $x=56$ or $x=57$ or $x=58$ or $x=59$ or $x=60$ or $x=61$ or $x=62$ or $x=63$.

(28) Let $S$ be a non empty set and $x_{1}, x_{2}, x_{3}, x_{4}, x_{5}, x_{6}, x_{7}, x_{8}$ be elements of $S$. Then there exists a finite sequence $s$ of elements of $S$ such that $s$ is 8-element and $s(1)=x_{1}$ and $s(2)=x_{2}$ and $s(3)=x_{3}$ and $s(4)=x_{4}$ and $s(5)=x_{5}$ and $s(6)=x_{6}$ and $s(7)=x_{7}$ and $s(8)=x_{8}$.

(29) Let $S$ be a non empty set and $x_{1}, x_{2}, x_{3}, x_{4}, x_{5}, x_{6}, x_{7}, x_{8}, x_{9}, x_{10}, x_{11}$, $x_{12}, x_{13}, x_{14}, x_{15}, x_{16}$ be elements of $S$. Then there exists a finite sequence $s$ of elements of $S$ such that

$s$ is 16-element and $s(1)=x_{1}$ and $s(2)=x_{2}$ and $s(3)=x_{3}$ and $s(4)=x_{4}$ and $s(5)=x_{5}$ and $s(6)=x_{6}$ and $s(7)=x_{7}$ and $s(8)=x_{8}$ and $s(9)=x_{9}$ and $s(10)=x_{10}$ and $s(11)=x_{11}$ and $s(12)=x_{12}$ and $s(13)=x_{13}$ and $s(14)=x_{14}$ and $s(15)=x_{15}$ and $s(16)=x_{16}$.

(30) Let $S$ be a non empty set and $x_{1}, x_{2}, x_{3}, x_{4}, x_{5}, x_{6}, x_{7}, x_{8}, x_{9}, x_{10}, x_{11}$, $x_{12}, x_{13}, x_{14}, x_{15}, x_{16}, x_{17}, x_{18}, x_{19}, x_{20}, x_{21}, x_{22}, x_{23}, x_{24}, x_{25}, x_{26}, x_{27}$, $x_{28}, x_{29}, x_{30}, x_{31}, x_{32}$ be elements of $S$. Then there exists a finite sequence $s$ of elements of $S$ such that

$s$ is 32 -element and $s(1)=x_{1}$ and $s(2)=x_{2}$ and $s(3)=x_{3}$ and $s(4)=x_{4}$ and $s(5)=x_{5}$ and $s(6)=x_{6}$ and $s(7)=x_{7}$ and $s(8)=x_{8}$ and $s(9)=x_{9}$ and $s(10)=x_{10}$ and $s(11)=x_{11}$ and $s(12)=x_{12}$ and $s(13)=x_{13}$ and $s(14)=x_{14}$ and $s(15)=x_{15}$ and $s(16)=x_{16}$ and $s(17)=x_{17}$ 
and $s(18)=x_{18}$ and $s(19)=x_{19}$ and $s(20)=x_{20}$ and $s(21)=x_{21}$ and $s(22)=x_{22}$ and $s(23)=x_{23}$ and $s(24)=x_{24}$ and $s(25)=x_{25}$ and $s(26)=x_{26}$ and $s(27)=x_{27}$ and $s(28)=x_{28}$ and $s(29)=x_{29}$ and $s(30)=x_{30}$ and $s(31)=x_{31}$ and $s(32)=x_{32}$.

(31) Let $S$ be a non empty set and $x_{1}, x_{2}, x_{3}, x_{4}, x_{5}, x_{6}, x_{7}, x_{8}, x_{9}, x_{10}, x_{11}$, $x_{12}, x_{13}, x_{14}, x_{15}, x_{16}, x_{17}, x_{18}, x_{19}, x_{20}, x_{21}, x_{22}, x_{23}, x_{24}, x_{25}, x_{26}, x_{27}$, $x_{28}, x_{29}, x_{30}, x_{31}, x_{32}, x_{33}, x_{34}, x_{35}, x_{36}, x_{37}, x_{38}, x_{39}, x_{40}, x_{41}, x_{42}, x_{43}$, $x_{44}, x_{45}, x_{46}, x_{47}, x_{48}$ be elements of $S$. Then there exists a finite sequence $s$ of elements of $S$ such that

$s$ is 48-element and $s(1)=x_{1}$ and $s(2)=x_{2}$ and $s(3)=x_{3}$ and $s(4)=x_{4}$ and $s(5)=x_{5}$ and $s(6)=x_{6}$ and $s(7)=x_{7}$ and $s(8)=x_{8}$ and $s(9)=x_{9}$ and $s(10)=x_{10}$ and $s(11)=x_{11}$ and $s(12)=x_{12}$ and $s(13)=x_{13}$ and $s(14)=x_{14}$ and $s(15)=x_{15}$ and $s(16)=x_{16}$ and $s(17)=x_{17}$ and $s(18)=x_{18}$ and $s(19)=x_{19}$ and $s(20)=x_{20}$ and $s(21)=x_{21}$ and $s(22)=x_{22}$ and $s(23)=x_{23}$ and $s(24)=x_{24}$ and $s(25)=x_{25}$ and $s(26)=x_{26}$ and $s(27)=x_{27}$ and $s(28)=x_{28}$ and $s(29)=x_{29}$ and $s(30)=x_{30}$ and $s(31)=x_{31}$ and $s(32)=x_{32}$ and $s(33)=x_{33}$ and $s(34)=x_{34}$ and $s(35)=x_{35}$ and $s(36)=x_{36}$ and $s(37)=x_{37}$ and $s(38)=x_{38}$ and $s(39)=x_{39}$ and $s(40)=x_{40}$ and $s(41)=x_{41}$ and $s(42)=x_{42}$ and $s(43)=x_{43}$ and $s(44)=x_{44}$ and $s(45)=x_{45}$ and $s(46)=x_{46}$ and $s(47)=x_{47}$ and $s(48)=x_{48}$.

(32) Let $S$ be a non empty set and $x_{1}, x_{2}, x_{3}, x_{4}, x_{5}, x_{6}, x_{7}, x_{8}, x_{9}, x_{10}, x_{11}$, $x_{12}, x_{13}, x_{14}, x_{15}, x_{16}, x_{17}, x_{18}, x_{19}, x_{20}, x_{21}, x_{22}, x_{23}, x_{24}, x_{25}, x_{26}, x_{27}$, $x_{28}, x_{29}, x_{30}, x_{31}, x_{32}, x_{33}, x_{34}, x_{35}, x_{36}, x_{37}, x_{38}, x_{39}, x_{40}, x_{41}, x_{42}, x_{43}$, $x_{44}, x_{45}, x_{46}, x_{47}, x_{48}, x_{49}, x_{50}, x_{51}, x_{52}, x_{53}, x_{54}, x_{55}, x_{56}$ be elements of $S$. Then there exists a finite sequence $s$ of elements of $S$ such that

$s$ is 56-element and $s(1)=x_{1}$ and $s(2)=x_{2}$ and $s(3)=x_{3}$ and $s(4)=x_{4}$ and $s(5)=x_{5}$ and $s(6)=x_{6}$ and $s(7)=x_{7}$ and $s(8)=x_{8}$ and $s(9)=x_{9}$ and $s(10)=x_{10}$ and $s(11)=x_{11}$ and $s(12)=x_{12}$ and $s(13)=x_{13}$ and $s(14)=x_{14}$ and $s(15)=x_{15}$ and $s(16)=x_{16}$ and $s(17)=x_{17}$ and $s(18)=x_{18}$ and $s(19)=x_{19}$ and $s(20)=x_{20}$ and $s(21)=x_{21}$ and $s(22)=x_{22}$ and $s(23)=x_{23}$ and $s(24)=x_{24}$ and $s(25)=x_{25}$ and $s(26)=x_{26}$ and $s(27)=x_{27}$ and $s(28)=x_{28}$ and $s(29)=x_{29}$ and $s(30)=x_{30}$ and $s(31)=x_{31}$ and $s(32)=x_{32}$ and $s(33)=x_{33}$ and $s(34)=x_{34}$ and $s(35)=x_{35}$ and $s(36)=x_{36}$ and $s(37)=x_{37}$ and $s(38)=x_{38}$ and $s(39)=x_{39}$ and $s(40)=x_{40}$ and $s(41)=x_{41}$ and $s(42)=x_{42}$ and $s(43)=x_{43}$ and $s(44)=x_{44}$ and $s(45)=x_{45}$ and $s(46)=x_{46}$ and $s(47)=x_{47}$ and $s(48)=x_{48}$ and $s(49)=x_{49}$ and $s(50)=x_{50}$ and $s(51)=x_{51}$ and $s(52)=x_{52}$ and $s(53)=x_{53}$ and $s(54)=x_{54}$ and $s(55)=x_{55}$ and $s(56)=x_{56}$.

(33) Let $S$ be a non empty set and $x_{1}, x_{2}, x_{3}, x_{4}, x_{5}, x_{6}, x_{7}, x_{8}, x_{9}, x_{10}, x_{11}$, 
$x_{12}, x_{13}, x_{14}, x_{15}, x_{16}, x_{17}, x_{18}, x_{19}, x_{20}, x_{21}, x_{22}, x_{23}, x_{24}, x_{25}, x_{26}, x_{27}$ $x_{28}, x_{29}, x_{30}, x_{31}, x_{32}, x_{33}, x_{34}, x_{35}, x_{36}, x_{37}, x_{38}, x_{39}, x_{40}, x_{41}, x_{42}, x_{43}$, $x_{44}, x_{45}, x_{46}, x_{47}, x_{48}, x_{49}, x_{50}, x_{51}, x_{52}, x_{53}, x_{54}, x_{55}, x_{56}, x_{57}, x_{58}, x_{59}$, $x_{60}, x_{61}, x_{62}, x_{63}, x_{64}$ be elements of $S$. Then there exists a finite sequence $s$ of elements of $S$ such that

$s$ is 64-element and $s(1)=x_{1}$ and $s(2)=x_{2}$ and $s(3)=x_{3}$ and $s(4)=x_{4}$ and $s(5)=x_{5}$ and $s(6)=x_{6}$ and $s(7)=x_{7}$ and $s(8)=x_{8}$ and $s(9)=x_{9}$ and $s(10)=x_{10}$ and $s(11)=x_{11}$ and $s(12)=x_{12}$ and $s(13)=x_{13}$ and $s(14)=x_{14}$ and $s(15)=x_{15}$ and $s(16)=x_{16}$ and $s(17)=x_{17}$ and $s(18)=x_{18}$ and $s(19)=x_{19}$ and $s(20)=x_{20}$ and $s(21)=x_{21}$ and $s(22)=x_{22}$ and $s(23)=x_{23}$ and $s(24)=x_{24}$ and $s(25)=x_{25}$ and $s(26)=x_{26}$ and $s(27)=x_{27}$ and $s(28)=x_{28}$ and $s(29)=x_{29}$ and $s(30)=x_{30}$ and $s(31)=x_{31}$ and $s(32)=x_{32}$ and $s(33)=x_{33}$ and $s(34)=x_{34}$ and $s(35)=x_{35}$ and $s(36)=x_{36}$ and $s(37)=x_{37}$ and $s(38)=x_{38}$ and $s(39)=x_{39}$ and $s(40)=x_{40}$ and $s(41)=x_{41}$ and $s(42)=x_{42}$ and $s(43)=x_{43}$ and $s(44)=x_{44}$ and $s(45)=x_{45}$ and $s(46)=x_{46}$ and $s(47)=x_{47}$ and $s(48)=x_{48}$ and $s(49)=x_{49}$ and $s(50)=x_{50}$ and $s(51)=x_{51}$ and $s(52)=x_{52}$ and $s(53)=x_{53}$ and $s(54)=x_{54}$ and $s(55)=x_{55}$ and $s(56)=x_{56}$ and $s(57)=x_{57}$ and $s(58)=x_{58}$ and $s(59)=x_{59}$ and $s(60)=x_{60}$ and $s(61)=x_{61}$ and $s(62)=x_{62}$ and $s(63)=x_{63}$ and $s(64)=x_{64}$.

Let $n$ be a non empty natural number and let $i$ be an element of $n$. We introduce ntoSeg $i$ as a synonym of $\operatorname{succ} i$.

Let $n$ be a non empty natural number and let $i$ be an element of $n$. Then ntoSeg $i$ is an element of $\operatorname{Seg} n$.

Let $n$ be a non empty natural number and let $f$ be a function from $n$ into $\operatorname{Seg} n$. We say that $f$ is NtoSeg if and only if:

(Def. 5) For every element $i$ of $n$ holds $f(i)=$ ntoSeg $i$.

Let $n$ be a non empty natural number. One can check that there exists a function from $n$ into $\operatorname{Seg} n$ which is NtoSeg.

Let $n$ be a non empty natural number. Observe that every function from $n$ into $\operatorname{Seg} n$ is bijective and NtoSeg.

We now state two propositions:

(34) Let $n$ be a non empty natural number, $f$ be an NtoSeg function from $n$ into Seg $n$, and $i$ be a natural number. If $i<n$, then $f(i)=i+1$ and $i \in \operatorname{dom} f$.

(35) Let $S$ be a non empty set and $x_{1}, x_{2}, x_{3}, x_{4}, x_{5}, x_{6}, x_{7}, x_{8}, x_{9}, x_{10}, x_{11}$, $x_{12}, x_{13}, x_{14}, x_{15}, x_{16}, x_{17}, x_{18}, x_{19}, x_{20}, x_{21}, x_{22}, x_{23}, x_{24}, x_{25}, x_{26}, x_{27}$ $x_{28}, x_{29}, x_{30}, x_{31}, x_{32}, x_{33}, x_{34}, x_{35}, x_{36}, x_{37}, x_{38}, x_{39}, x_{40}, x_{41}, x_{42}, x_{43}$, $x_{44}, x_{45}, x_{46}, x_{47}, x_{48}, x_{49}, x_{50}, x_{51}, x_{52}, x_{53}, x_{54}, x_{55}, x_{56}, x_{57}, x_{58}, x_{59}$, $x_{60}, x_{61}, x_{62}, x_{63}, x_{64}$ be elements of $S$. Then there exists a function $f$ 
from 64 into $S$ such that

$f(0)=x_{1}$ and $f(1)=x_{2}$ and $f(2)=x_{3}$ and $f(3)=x_{4}$ and $f(4)=x_{5}$ and $f(5)=x_{6}$ and $f(6)=x_{7}$ and $f(7)=x_{8}$ and $f(8)=x_{9}$ and $f(9)=x_{10}$ and $f(10)=x_{11}$ and $f(11)=x_{12}$ and $f(12)=x_{13}$ and $f(13)=x_{14}$ and $f(14)=x_{15}$ and $f(15)=x_{16}$ and $f(16)=x_{17}$ and $f(17)=x_{18}$ and $f(18)=x_{19}$ and $f(19)=x_{20}$ and $f(20)=x_{21}$ and $f(21)=x_{22}$ and $f(22)=x_{23}$ and $f(23)=x_{24}$ and $f(24)=x_{25}$ and $f(25)=x_{26}$ and $f(26)=x_{27}$ and $f(27)=x_{28}$ and $f(28)=x_{29}$ and $f(29)=x_{30}$ and $f(30)=x_{31}$ and $f(31)=x_{32}$ and $f(32)=x_{33}$ and $f(33)=x_{34}$ and $f(34)=x_{35}$ and $f(35)=x_{36}$ and $f(36)=x_{37}$ and $f(37)=x_{38}$ and $f(38)=x_{39}$ and $f(39)=x_{40}$ and $f(40)=x_{41}$ and $f(41)=x_{42}$ and $f(42)=x_{43}$ and $f(43)=x_{44}$ and $f(44)=x_{45}$ and $f(45)=x_{46}$ and $f(46)=x_{47}$ and $f(47)=x_{48}$ and $f(48)=x_{49}$ and $f(49)=x_{50}$ and $f(50)=x_{51}$ and $f(51)=x_{52}$ and $f(52)=x_{53}$ and $f(53)=x_{54}$ and $f(54)=x_{55}$ and $f(55)=x_{56}$ and $f(56)=x_{57}$ and $f(57)=x_{58}$ and $f(58)=x_{59}$ and $f(59)=x_{60}$ and $f(60)=x_{61}$ and $f(61)=x_{62}$ and $f(62)=x_{63}$ and $f(63)=x_{64}$.

\section{S-Boxes}

The function DES-SBOX1 from 64 into 16 is defined by the conditions (Def. 6).

(Def. 6$)($ DES-SBOX1 $)(0)=14$ and $($ DES-SBOX1 $)(1)=4$ and $($ DES-SBOX1 $)(2)=$ 13 and $($ DES-SBOX 1$)(3)=1$ and $($ DES-SBOX 1$)(4)=2$ and $($ DES-SBOX1) $(5)=15$ and $($ DES-SBOX1)(6) $=11$ and $($ DES-SBOX 1$)(7)=8$ and $($ DES-SBOX 1$)(8)=3$ and $($ DES-SBOX 1$)(9)=$ 10 and $($ DES-SBOX 1$)(10)=6$ and $($ DES-SBOX 1$)(11)=12$ and $($ DES-SBOX1)(12) $=5$ and $($ DES-SBOX1)(13) $=9$ and $($ DES-SBOX 1$)(14)=0$ and $($ DES-SBOX 1$)(15)=7$ and $($ DES-SBOX1 $)(16)=$ 0 and $($ DES-SBOX1)(17) $=15$ and $($ DES-SBOX1) $(18)=7$ and $($ DES-SBOX1)(19) $=4$ and $($ DES-SBOX1) $(20)=14$ and $($ DES-SBOX 1$)(21)=2$ and $($ DES-SBOX1) $(22)=13$ and $($ DES-SBOX1 $)(23)=$ 1 and $($ DES-SBOX 1$)(24)=10$ and $($ DES-SBOX1) $(25)=6$ and $($ DES-SBOX1 $)(26)=12$ and $($ DES-SBOX1) $(27)=11$ and $($ DES-SBOX1) $(28)=9$ and $($ DES-SBOX1) $(29)=5$ and $($ DES-SBOX 1$)(30)=3$ and $($ DES-SBOX 1$)(31)=8$ and $($ DES-SBOX1 $)(32)=$ 4 and $($ DES-SBOX1)(33) $=1$ and $($ DES-SBOX1) $(34)=14$ and $($ DES-SBOX1) $(35)=8$ and $($ DES-SBOX1) $(36)=13$ and $($ DES-SBOX1 $)(37)=6$ and $($ DES-SBOX1 $)(38)=2$ and $($ DES-SBOX1 $)(39)=$ 11 and $($ DES-SBOX1)(40) $=15$ and $($ DES-SBOX1) $(41)=12$ and $($ DES-SBOX1) $(42)=9$ and $($ DES-SBOX1) $(43)=7$ and 
$($ DES-SBOX 1$)(44)=3$ and $($ DES-SBOX 1$)(45)=10$ and $($ DES-SBOX1 $)(46)=$ 5 and $($ DES-SBOX1)(47) $=0$ and $($ DES-SBOX1) $(48)=15$ and $($ DES-SBOX1) $(49)=12$ and $($ DES-SBOX1)(50) $=8$ and $($ DES-SBOX1) $(51)=2$ and $($ DES-SBOX1) $(52)=4$ and $($ DES-SBOX1) $(53)=$ 9 and $($ DES-SBOX1) $(54)=1$ and $($ DES-SBOX1) $(55)=7$ and $($ DES-SBOX1) $(56)=5$ and $($ DES-SBOX1)(57) $=11$ and $($ DES-SBOX 1$)(58)=3$ and $($ DES-SBOX1) $(59)=14$ and $($ DES-SBOX1) $(60)=$ 10 and $($ DES-SBOX1)(61) $=0$ and $($ DES-SBOX1)(62) $=6$ and $($ DES-SBOX1) $(63)=13$.

The function DES-SBOX2 from 64 into 16 is defined by the conditions (Def. 7).

(Def. 7$)($ DES-SBOX2 $)(0)=15$ and $($ DES-SBOX2 $)(1)=1$ and $($ DES-SBOX2 $)(2)=$ 8 and $($ DES-SBOX2) $(3)=14$ and $($ DES-SBOX2) $(4)=6$ and $($ DES-SBOX2) $(5)=11$ and $($ DES-SBOX2) $(6)=3$ and $($ DES-SBOX2 $)(7)=4$ and $($ DES-SBOX2 $)(8)=9$ and $($ DES-SBOX2 $)(9)=$ 7 and $($ DES-SBOX2) $(10)=2$ and $($ DES-SBOX2) $(11)=13$ and $($ DES-SBOX2) $(12)=12$ and $($ DES-SBOX2) $(13)=0$ and $($ DES-SBOX2 $)(14)=5$ and $($ DES-SBOX2 $)(15)=10$ and $($ DES-SBOX2 $)(16)=$ 3 and $($ DES-SBOX2) $(17)=13$ and $($ DES-SBOX2) $(18)=4$ and $($ DES-SBOX2) $(19)=7$ and $($ DES-SBOX2) $(20)=15$ and $($ DES-SBOX2 $)(21)=2$ and $($ DES-SBOX2 $)(22)=8$ and $($ DES-SBOX2 $)(23)=$ 14 and $($ DES-SBOX2) $(24)=12$ and $($ DES-SBOX2) $(25)=0$ and $($ DES-SBOX2) $(26)=1$ and $($ DES-SBOX2) $(27)=10$ and $($ DES-SBOX2 $)(28)=6$ and $($ DES-SBOX2 $)(29)=9$ and $($ DES-SBOX2 $)(30)=$ 11 and $($ DES-SBOX2) $(31)=5$ and $($ DES-SBOX2) $(32)=0$ and $($ DES-SBOX2) $(33)=14$ and $($ DES-SBOX2) $(34)=7$ and $($ DES-SBOX2) $(35)=11$ and $($ DES-SBOX2) $(36)=10$ and $($ DES-SBOX2 $)(37)=4$ and $($ DES-SBOX2 $)(38)=13$ and $($ DES-SBOX2 $)(39)=$ 1 and $($ DES-SBOX2) $(40)=5$ and $($ DES-SBOX2) $(41)=8$ and $($ DES-SBOX2) $(42)=12$ and (DES-SBOX2)(43) $=6$ and $($ DES-SBOX2) $(44)=9$ and $($ DES-SBOX2 $)(45)=3$ and $($ DES-SBOX2) $(46)=$ 2 and $($ DES-SBOX2) $(47)=15$ and $($ DES-SBOX2) $(48)=13$ and $($ DES-SBOX2) $(49)=8$ and $($ DES-SBOX2) $(50)=10$ and $($ DES-SBOX2 $)(51)=1$ and $($ DES-SBOX2 $)(52)=3$ and $($ DES-SBOX2) $(53)=$ 15 and $($ DES-SBOX2) $(54)=4$ and $($ DES-SBOX2) $(55)=2$ and $($ DES-SBOX2) $(56)=11$ and $($ DES-SBOX2) $(57)=6$ and $($ DES-SBOX2 $)(58)=7$ and $($ DES-SBOX2 $)(59)=12$ and $($ DES-SBOX2 $)(60)=$ 0 and $($ DES-SBOX2)(61) $=5$ and $($ DES-SBOX2)(62) $=14$ and $($ DES-SBOX2 $)(63)=9$.

The function DES-SBOX3 from 64 into 16 is defined by the conditions (Def. 8). 
(Def. 8$)($ DES-SBOX3) $(0)=10$ and $($ DES-SBOX3 $)(1)=0$ and $($ DES-SBOX3 $)(2)=$ 9 and $($ DES-SBOX3) $(3)=14$ and $($ DES-SBOX3) $(4)=6$ and $($ DES-SBOX 3$)(5)=3$ and $($ DES-SBOX3 $)(6)=15$ and $($ DES-SBOX3 $)(7)=5$ and $($ DES-SBOX3 $)(8)=1$ and $($ DES-SBOX3 $)(9)=$ 13 and $($ DES-SBOX3)(10) $=12$ and $($ DES-SBOX3) $(11)=7$ and $($ DES-SBOX3) $(12)=11$ and $($ DES-SBOX3) $(13)=4$ and $($ DES-SBOX3) $(14)=2$ and $($ DES-SBOX3 $)(15)=8$ and $($ DES-SBOX3 $)(16)=$ 13 and $($ DES-SBOX3) $(17)=7$ and $($ DES-SBOX3) $(18)=0$ and $($ DES-SBOX3) $(19)=9$ and $($ DES-SBOX3) $(20)=3$ and $($ DES-SBOX3 $)(21)=4$ and $($ DES-SBOX3 $)(22)=6$ and $($ DES-SBOX3 $)(23)=$ 10 and $($ DES-SBOX3) $(24)=2$ and $($ DES-SBOX3) $(25)=8$ and $($ DES-SBOX3) $(26)=5$ and $($ DES-SBOX3) $(27)=14$ and $($ DES-SBOX3) $(28)=12$ and $($ DES-SBOX3) $(29)=11$ and $($ DES-SBOX3 $)(30)=15$ and $($ DES-SBOX3 $)(31)=1$ and $($ DES-SBOX3 $)(32)=$ 13 and $($ DES-SBOX3) $(33)=6$ and $($ DES-SBOX3) $(34)=4$ and $($ DES-SBOX3) $(35)=9$ and $($ DES-SBOX3) $(36)=8$ and $($ DES-SBOX3 $)(37)=15$ and $($ DES-SBOX3 $)(38)=3$ and $($ DES-SBOX3 $)(39)=$ 0 and $($ DES-SBOX3) $(40)=11$ and $($ DES-SBOX3) $(41)=1$ and $($ DES-SBOX3) $(42)=2$ and $($ DES-SBOX3)(43) $=12$ and $($ DES-SBOX3 $)(44)=5$ and $($ DES-SBOX3 $)(45)=10$ and $($ DES-SBOX3) $(46)=$ 14 and $($ DES-SBOX3) $(47)=7$ and $($ DES-SBOX3) $(48)=1$ and $($ DES-SBOX3) $(49)=10$ and $($ DES-SBOX3) $(50)=13$ and $($ DES-SBOX3) $(51)=0$ and $($ DES-SBOX3) $(52)=6$ and $($ DES-SBOX3) $(53)=9$ and $($ DES-SBOX 3$)(54)=8$ and $($ DES-SBOX3) $(55)=$ 7 and $($ DES-SBOX3) $(56)=4$ and $($ DES-SBOX3) $(57)=15$ and $($ DES-SBOX3) $(58)=14$ and $($ DES-SBOX3)(59) $=3$ and $($ DES-SBOX3 $)(60)=11$ and $($ DES-SBOX3 $)(61)=5$ and $($ DES-SBOX3 $)(62)=$ 2 and $($ DES-SBOX3) $(63)=12$.

The function DES-SBOX4 from 64 into 16 is defined by the conditions (Def. 9).

(Def. 9) $($ DES-SBOX4 $)(0)=7$ and $($ DES-SBOX4 $)(1)=13$ and $($ DES-SBOX4 $)(2)=$ 14 and $($ DES-SBOX 4$)(3)=3$ and $($ DES-SBOX4) $(4)=0$ and $($ DES-SBOX 4$)(5)=6$ and $($ DES-SBOX 4$)(6)=9$ and $($ DES-SBOX 4$)(7)=$ 10 and $($ DES-SBOX 4$)(8)=1$ and $($ DES-SBOX 4$)(9)=2$ and $($ DES-SBOX4) $(10)=8$ and $($ DES-SBOX4) $(11)=5$ and $($ DES-SBOX 4$)(12)=11$ and $($ DES-SBOX4) $(13)=12$ and $($ DES-SBOX 4$)(14)=4$ and $($ DES-SBOX 4$)(15)=15$ and $($ DES-SBOX 4$)(16)=$ 13 and $($ DES-SBOX 4$)(17)=8$ and $($ DES-SBOX4) $(18)=11$ and $($ DES-SBOX4) $(19)=5$ and $($ DES-SBOX4) $(20)=6$ and $($ DES-SBOX 4$)(21)=15$ and $($ DES-SBOX4 $)(22)=0$ and $($ DES-SBOX 4$)(23)=$ 3 and $($ DES-SBOX 4$)(24)=4$ and $($ DES-SBOX4 $)(25)=7$ 
and $($ DES-SBOX4) $(26)=2$ and $($ DES-SBOX4) $(27)=12$ and $($ DES-SBOX 4$)(28)=1$ and $($ DES-SBOX4 $)(29)=10$ and $($ DES-SBOX 4$)(30)=$ 14 and $($ DES-SBOX 4$)(31)=9$ and $($ DES-SBOX 4$)(32)=10$ and $($ DES-SBOX4) $(33)=6$ and $($ DES-SBOX4) $(34)=9$ and $($ DES-SBOX 4$)(35)=0$ and $($ DES-SBOX 4$)(36)=12$ and $($ DES-SBOX 4$)(37)=$ 11 and $($ DES-SBOX4) $(38)=7$ and $($ DES-SBOX4) $(39)=13$ and $($ DES-SBOX4) $(40)=15$ and $($ DES-SBOX4) $(41)=1$ and $($ DES-SBOX 4$)(42)=3$ and $($ DES-SBOX4 $)(43)=14$ and $($ DES-SBOX 4$)(44)=$ 5 and $($ DES-SBOX 4$)(45)=2$ and $($ DES-SBOX4) $(46)=8$ and $($ DES-SBOX4) $(47)=4$ and $($ DES-SBOX4) $(48)=3$ and $($ DES-SBOX 4$)(49)=15$ and $($ DES-SBOX 4$)(50)=0$ and $($ DES-SBOX 4$)(51)=$ 6 and $($ DES-SBOX 4$)(52)=10$ and $($ DES-SBOX4) $(53)=1$ and $($ DES-SBOX 4$)(54)=13$ and $($ DES-SBOX4) $(55)=8$ and $($ DES-SBOX4 $)(56)=9$ and $($ DES-SBOX 4$)(57)=4$ and $($ DES-SBOX4 $)(58)=$ 5 and $($ DES-SBOX4) $(59)=11$ and $($ DES-SBOX4) $(60)=12$ and $($ DES-SBOX4) $(61)=7$ and $($ DES-SBOX4) $(62)=2$ and $(\mathrm{DES}-\mathrm{SBOX} 4)(63)=14$.

The function DES-SBOX5 from 64 into 16 is defined by the conditions (Def. 10).

(Def. 10) $($ DES-SBOX5 $)(0)=2$ and $($ DES-SBOX5 $)(1)=12$ and $($ DES-SBOX5 $)(2)=$ 4 and $($ DES-SBOX5)(3) $=1$ and $($ DES-SBOX5) $(4)=7$ and $($ DES-SBOX 5$)(5)=10$ and $($ DES-SBOX 5$)(6)=11$ and $($ DES-SBOX 5$)(7)=$ 6 and $($ DES-SBOX5)(8) $=8$ and $($ DES-SBOX5) $(9)=5$ and $($ DES-SBOX 5$)(10)=3$ and $($ DES-SBOX 5$)(11)=15$ and $($ DES-SBOX5 $)(12)=$ 13 and $($ DES-SBOX5) $(13)=0$ and $($ DES-SBOX5) $(14)=14$ and $($ DES-SBOX5) $(15)=9$ and $($ DES-SBOX5) $(16)=14$ and $($ DES-SBOX $)(17)=11$ and $($ DES-SBOX5 $)(18)=2$ and $($ DES-SBOX5 $)(19)=$ 12 and $($ DES-SBOX5) $(20)=4$ and $($ DES-SBOX5) $(21)=7$ and $($ DES-SBOX5) $(22)=13$ and $($ DES-SBOX5) $(23)=1$ and $($ DES-SBOX5 $)(24)=5$ and $($ DES-SBOX 5$)(25)=0$ and $($ DES-SBOX5 $)(26)=$ 15 and $($ DES-SBOX5) $(27)=10$ and $($ DES-SBOX5) $(28)=3$ and $($ DES-SBOX5) $(29)=9$ and $($ DES-SBOX5)(30) $=8$ and $($ DES-SBOX5 $)(31)=6$ and $($ DES-SBOX5 $)(32)=4$ and $($ DES-SBOX5 $)(33)=$ 2 and $($ DES-SBOX5) $(34)=1$ and $($ DES-SBOX5) $(35)=11$ and $($ DES-SBOX5) $(36)=10$ and $($ DES-SBOX5) $(37)=13$ and $($ DES-SBOX5) $(38)=7$ and $($ DES-SBOX5) $(39)=8$ and $($ DES-SBOX $)(40)=15$ and $($ DES-SBOX 5$)(41)=9$ and $($ DES-SBOX 5$)(42)=$ 12 and $($ DES-SBOX 5$)(43)=5$ and $($ DES-SBOX5) $(44)=6$ and $($ DES-SBOX5) $(45)=3$ and $($ DES-SBOX5) $(46)=0$ and $($ DES-SBOX 5$)(47)=14$ and $($ DES-SBOX5) $(48)=11$ and $($ DES-SBOX 5$)(49)=8$ and $($ DES-SBOX5 $)(50)=12$ and $($ DES-SBOX5) $(51)=$ 
7 and $($ DES-SBOX5)(52) $=1$ and $($ DES-SBOX5)(53) $=14$ and $($ DES-SBOX5) $(54)=2$ and $($ DES-SBOX5) $(55)=13$ and $($ DES-SBOX5) $(56)=6$ and $($ DES-SBOX5 $)(57)=15$ and $($ DES-SBOX5) $(58)=$ 0 and $($ DES-SBOX5) $(59)=9$ and $($ DES-SBOX5) $(60)=10$ and $($ DES-SBOX5) $(61)=4$ and $($ DES-SBOX5) $(62)=5$ and $($ DES-SBOX5) $(63)=3$.

The function DES-SBOX6 from 64 into 16 is defined by the conditions (Def. 11).

(Def. 11) $($ DES-SBOX6) $(0)=12$ and $($ DES-SBOX6) $(1)=1$ and $($ DES-SBOX6) $(2)=$ 10 and $($ DES-SBOX6) $(3)=15$ and $($ DES-SBOX6) $(4)=9$ and $($ DES-SBOX6) $(5)=2$ and $($ DES-SBOX6) $(6)=6$ and $($ DES-SBOX 6$)(7)=8$ and $($ DES-SBOX6 $)(8)=0$ and $($ DES-SBOX6) $(9)=$ 13 and $($ DES-SBOX6) $(10)=3$ and $($ DES-SBOX6) $(11)=4$ and $($ DES-SBOX6) $(12)=14$ and $($ DES-SBOX6) $(13)=7$ and $($ DES-SBOX6) $(14)=5$ and $($ DES-SBOX6 $)(15)=11$ and $($ DES-SBOX6 $)(16)=$ 10 and $($ DES-SBOX6) $(17)=15$ and $($ DES-SBOX6) $(18)=4$ and $($ DES-SBOX6) $(19)=2$ and $($ DES-SBOX6) $(20)=7$ and $($ DES-SBOX6) $(21)=12$ and $($ DES-SBOX6) $(22)=9$ and $($ DES-SBOX6) $(23)=$ 5 and $($ DES-SBOX6) $(24)=6$ and $($ DES-SBOX6) $(25)=1$ and $($ DES-SBOX6) $(26)=13$ and $($ DES-SBOX6) $(27)=14$ and $($ DES-SBOX6) $(28)=0$ and $($ DES-SBOX6) $(29)=11$ and $($ DES-SBOX6) $(30)=3$ and $($ DES-SBOX6) $(31)=8$ and $($ DES-SBOX6) $(32)=$ 9 and $($ DES-SBOX6) $(33)=14$ and $($ DES-SBOX6) $(34)=15$ and $($ DES-SBOX6) $(35)=5$ and $($ DES-SBOX6) $(36)=2$ and $($ DES-SBOX6) $(37)=8$ and $($ DES-SBOX6) $(38)=12$ and $($ DES-SBOX6) $(39)=$ 3 and $($ DES-SBOX6) $(40)=7$ and $($ DES-SBOX6) $(41)=0$ and $($ DES-SBOX6) $(42)=4$ and $($ DES-SBOX6) $(43)=10$ and $($ DES-SBOX6) $(44)=1$ and $($ DES-SBOX6) $(45)=13$ and $($ DES-SBOX6 $)(46)=$ 11 and $($ DES-SBOX6) $(47)=6$ and $($ DES-SBOX6) $(48)=4$ and $($ DES-SBOX6) $(49)=3$ and $($ DES-SBOX6) $(50)=2$ and $($ DES-SBOX6) $(51)=12$ and $($ DES-SBOX6) $(52)=9$ and $($ DES-SBOX6) $(53)=$ 5 and $($ DES-SBOX6) $(54)=15$ and $($ DES-SBOX6) $(55)=10$ and $($ DES-SBOX6) $(56)=11$ and $($ DES-SBOX6) $(57)=14$ and $($ DES-SBOX6) $(58)=1$ and $($ DES-SBOX6) $(59)=7$ and $($ DES-SBOX6) $(60)=6$ and $($ DES-SBOX6) $(61)=0$ and $($ DES-SBOX6) $(62)=$ 8 and $($ DES-SBOX6) $(63)=13$.

The function DES-SBOX7 from 64 into 16 is defined by the conditions (Def. 12).

(Def. 12) $($ DES-SBOX7 $)(0)=4$ and $($ DES-SBOX7 $)(1)=11$ and $($ DES-SBOX7 $)(2)=$ 2 and $($ DES-SBOX7) $(3)=14$ and $($ DES-SBOX7)(4) $=15$ and $($ DES-SBOX 7$)(5)=0$ and $($ DES-SBOX7 $)(6)=8$ and $($ DES-SBOX7 $)(7)=$ 
13 and $($ DES-SBOX 7$)(8)=3$ and $($ DES-SBOX7) $(9)=12$ and $($ DES-SBOX7) $(10)=9$ and $($ DES-SBOX7) $(11)=7$ and $($ DES-SBOX 7$)(12)=5$ and $($ DES-SBOX 7$)(13)=10$ and $($ DES-SBOX 7$)(14)=$ 6 and $($ DES-SBOX7) $(15)=1$ and $($ DES-SBOX7) $(16)=13$ and $($ DES-SBOX7) $(17)=0$ and $($ DES-SBOX7)(18) $=11$ and $($ DES-SBOX7 $)(19)=7$ and $($ DES-SBOX7 $)(20)=4$ and $($ DES-SBOX7) $(21)=$ 9 and $($ DES-SBOX7) $(22)=1$ and $($ DES-SBOX7) $(23)=10$ and $($ DES-SBOX7) $(24)=14$ and $($ DES-SBOX7) $(25)=3$ and $($ DES-SBOX 7$)(26)=5$ and $($ DES-SBOX7 $)(27)=12$ and $($ DES-SBOX7 $)(28)=$ 2 and $($ DES-SBOX7) $(29)=15$ and $($ DES-SBOX7) $(30)=8$ and $($ DES-SBOX7) $(31)=6$ and $($ DES-SBOX7) $(32)=1$ and $($ DES-SBOX 7$)(33)=4$ and $($ DES-SBOX7 $)(34)=11$ and $($ DES-SBOX7) $(35)=$ 13 and $($ DES-SBOX7) $(36)=12$ and $($ DES-SBOX7) $(37)=3$ and $($ DES-SBOX7)(38) $=7$ and $($ DES-SBOX7) $(39)=14$ and $($ DES-SBOX7) $(40)=10$ and $($ DES-SBOX7) $(41)=15$ and $($ DES-SBOX 7$)(42)=6$ and $($ DES-SBOX 7$)(43)=8$ and $($ DES-SBOX7 $)(44)=$ 0 and $($ DES-SBOX7) $(45)=5$ and $($ DES-SBOX7) $(46)=9$ and $($ DES-SBOX7) $(47)=2$ and $($ DES-SBOX7) $(48)=6$ and $($ DES-SBOX 7$)(49)=11$ and $($ DES-SBOX7) $(50)=13$ and $($ DES-SBOX7) $(51)=8$ and $($ DES-SBOX 7$)(52)=1$ and $($ DES-SBOX7 $)(53)=$ 4 and $($ DES-SBOX 7$)(54)=10$ and $($ DES-SBOX7) $(55)=7$ and $($ DES-SBOX7) $(56)=9$ and $($ DES-SBOX7) $(57)=5$ and $($ DES-SBOX 7$)(58)=0$ and $($ DES-SBOX7 $)(59)=15$ and $($ DES-SBOX7) $(60)=$ 14 and $($ DES-SBOX7)(61) $=2$ and $($ DES-SBOX7) $(62)=3$ and $(\mathrm{DES}-\mathrm{SBOX} 7)(63)=12$.

The function DES-SBOX8 from 64 into 16 is defined by the conditions (Def. 13).

$($ Def. 13$) \quad($ DES-SBOX8) $(0)=13$ and $($ DES-SBOX8) $(1)=2$ and $($ DES-SBOX8) $(2)=$ 8 and $($ DES-SBOX 8$)(3)=4$ and $($ DES-SBOX 8$)(4)=6$ and $($ DES-SBOX 8$)(5)=15$ and $($ DES-SBOX $)(6)=11$ and $($ DES-SBOX 8$)(7)=$ 1 and $($ DES-SBOX 8$)(8)=10$ and $($ DES-SBOX8) $(9)=9$ and $($ DES-SBOX8)(10) $=3$ and $($ DES-SBOX8)(11) $=14$ and $($ DES-SBOX 8$)(12)=5$ and $($ DES-SBOX 8$)(13)=0$ and $($ DES-SBOX8) $(14)=$ 12 and $($ DES-SBOX8) $(15)=7$ and $($ DES-SBOX8) $(16)=1$ and $($ DES-SBOX8) $(17)=15$ and $($ DES-SBOX8) $(18)=13$ and $($ DES-SBOX8)(19) $=8$ and $($ DES-SBOX8) $(20)=10$ and $($ DES-SBOX $)(21)=3$ and $($ DES-SBOX 8$)(22)=7$ and $($ DES-SBOX 8$)(23)=$ 4 and $($ DES-SBOX 8$)(24)=12$ and $($ DES-SBOX8) $(25)=5$ and $($ DES-SBOX8) $(26)=5$ and $($ DES-SBOX8) $(27)=11$ and $($ DES-SBOX 8$)(28)=0$ and $($ DES-SBOX8) $(29)=14$ and $($ DES-SBOX8) $(30)=$ 9 and $($ DES-SBOX $)(31)=2$ and $($ DES-SBOX8) $(32)=7$ 
and $($ DES-SBOX8)(33) $=11$ and $($ DES-SBOX8) $(34)=4$ and $($ DES-SBOX 8$)(35)=1$ and $($ DES-SBOX8) $(36)=9$ and $($ DES-SBOX8) $(37)=$ 12 and $($ DES-SBOX8) $(38)=14$ and $($ DES-SBOX8) $(39)=2$ and $($ DES-SBOX8) $(40)=0$ and $($ DES-SBOX8) $(41)=6$ and $($ DES-SBOX 8$)(42)=10$ and $($ DES-SBOX8) $(43)=13$ and $($ DES-SBOX 8$)(44)=15$ and $($ DES-SBOX 8$)(45)=3$ and $($ DES-SBOX 8$)(46)=$ 5 and $($ DES-SBOX 8$)(47)=8$ and $($ DES-SBOX 8$)(48)=2$ and $($ DES-SBOX8) $(49)=1$ and $($ DES-SBOX8) $(50)=14$ and $($ DES-SBOX 8$)(51)=7$ and $($ DES-SBOX 8$)(52)=4$ and $($ DES-SBOX8) $(53)=$ 10 and $($ DES-SBOX8) $(54)=8$ and $($ DES-SBOX8) $(55)=13$ and $($ DES-SBOX 8$)(56)=15$ and $($ DES-SBOX8) $(57)=12$ and $($ DES-SBOX8)(58) $=9$ and $($ DES-SBOX8) $(59)=0$ and $($ DES-SBOX 8$)(60)=3$ and $($ DES-SBOX8) $(61)=5$ and $($ DES-SBOX 8$)(62)=$ 6 and $($ DES-SBOX8) $(63)=11$.

\section{Initial Permutation}

Let $r$ be an element of Boolean ${ }^{64}$. The functor DES-IP $r$ yields an element of Boolean ${ }^{64}$ and is defined by the conditions (Def. 14).

(Def. 14) $($ DES-IP $r)(1)=r(58)$ and $($ DES-IP $r)(2)=r(50)$ and $($ DES-IP $r)(3)=$ $r(42)$ and $($ DES-IP $r)(4)=r(34)$ and $($ DES-IP $r)(5)=r(26)$ and $(\mathrm{DES}-\mathrm{IP} r)(6)=r(18)$ and $(\mathrm{DES}-\mathrm{IP} r)(7)=r(10)$ and $($ DES-IP $r)(8)=r(2)$ and $($ DES-IP $r)(9)=r(60)$ and $($ DES-IP $r)(10)=$ $r(52)$ and $($ DES-IP $r)(11)=r(44)$ and $($ DES-IP $r)(12)=r(36)$ and $($ DES-IP $r)(13)=r(28)$ and $($ DES-IP $r)(14)=r(20)$ and $($ DES-IP $r)(15)=r(12)$ and $($ DES-IP $r)(16)=r(4)$ and $($ DES-IP $r)(17)=$ $r(62)$ and $($ DES-IP $r)(18)=r(54)$ and $($ DES-IP $r)(19)=r(46)$ and $($ DES-IP $r)(20)=r(38)$ and $($ DES-IP $r)(21)=r(30)$ and $($ DES-IP $r)(22)=r(22)$ and $($ DES-IP $r)(23)=r(14)$ and $($ DES-IP $r)(24)=$ $r(6)$ and $(\mathrm{DES}-\mathrm{IP} r)(25)=r(64)$ and $(\mathrm{DES}-\mathrm{IP} r)(26)=r(56)$ and $(\mathrm{DES}-\mathrm{IP} r)(27)=r(48)$ and $(\mathrm{DES}-\mathrm{IP} r)(28)=r(40)$ and $($ DES-IP $r)(29)=r(32)$ and $($ DES-IP $r)(30)=r(24)$ and $($ DES-IP $r)(31)=$ $r(16)$ and $($ DES-IP $r)(32)=r(8)$ and $($ DES-IP $r)(33)=r(57)$ and $(\mathrm{DES}-\mathrm{IP} r)(34)=r(49)$ and $(\mathrm{DES}-\mathrm{IP} r)(35)=r(41)$ and $($ DES-IP $r)(36)=r(33)$ and $($ DES-IP $r)(37)=r(25)$ and $($ DES-IP $r)(38)=$ $r(17)$ and $(\mathrm{DES}-\mathrm{IP} r)(39)=r(9)$ and $(\mathrm{DES}-\mathrm{IP} r)(40)=r(1)$ and $($ DES-IP $r)(41)=r(59)$ and $($ DES-IP $r)(42)=r(51)$ and $($ DES-IP $r)(43)=r(43)$ and $($ DES-IP $r)(44)=r(35)$ and $($ DES-IP $r)(45)=$ $r(27)$ and (DES-IP $r)(46)=r(19)$ and $($ DES-IP $r)(47)=r(11)$ and $($ DES-IP $r)(48)=r(3)$ and $($ DES-IP $r)(49)=r(61)$ and $($ DES-IP $r)(50)=$ $r(53)$ and $($ DES-IP $r)(51)=r(45)$ and $($ DES-IP $r)(52)=r(37)$ 
and $(\mathrm{DES}-\mathrm{IP} r)(53)=r(29)$ and $(\mathrm{DES}-\mathrm{IP} r)(54)=r(21)$ and $($ DES-IP $r)(55)=r(13)$ and $($ DES-IP $r)(56)=r(5)$ and $($ DES-IP $r)(57)=$ $r(63)$ and $($ DES-IP $r)(58)=r(55)$ and $($ DES-IP $r)(59)=r(47)$ and $($ DES-IP $r)(60)=r(39)$ and $($ DES-IP $r)(61)=r(31)$ and $($ DES-IP $r)(62)=r(23)$ and $($ DES-IP $r)(63)=r(15)$ and $($ DES-IP $r)(64)=$ $r(7)$.

The function DES-PIP from Boolean ${ }^{64}$ into Boolean ${ }^{64}$ is defined by:

(Def. 15) For every element $i$ of Boolean ${ }^{64}$ holds (DES-PIP) $(i)=\operatorname{DES-IP~} i$.

Let $r$ be an element of Boolean ${ }^{64}$. The functor DES-IPINV $r$ yields an element of Boolean ${ }^{64}$ and is defined by the conditions (Def. 16).

$($ Def. 16) $($ DES-IPINV $r)(1)=r(40)$ and $($ DES-IPINV $r)(2)=r(8)$ and $(\mathrm{DES}-\mathrm{IPINV} r)(3)=r(48)$ and $(\mathrm{DES}-\mathrm{IPINV} r)(4)=r(16)$ and $(\mathrm{DES}-\mathrm{IPINV} r)(5)=r(56)$ and $(\mathrm{DES}-\mathrm{IPINV} r)(6)=r(24)$ and $(\mathrm{DES}-\mathrm{IPINV} r)(7)=r(64)$ and $(\mathrm{DES}-\mathrm{IPINV} r)(8)=r(32)$ and $(\mathrm{DES}-\mathrm{IPINV} r)(9)=r(39)$ and $(\mathrm{DES}-\mathrm{IPINV} r)(10)=r(7)$ and $(\mathrm{DES}-\mathrm{IPINV} r)(11)=r(47)$ and $(\mathrm{DES}-\mathrm{IPINV} r)(12)=r(15)$ and $($ DES-IPINV $r)(13)=r(55)$ and $($ DES-IPINV $r)(14)=r(23)$ and $($ DES-IPINV $r)(15)=r(63)$ and $($ DES-IPINV $r)(16)=r(31)$ and $(\mathrm{DES}-I P I N V r)(17)=r(38)$ and $($ DES-IPINV $r)(18)=r(6)$ and $($ DES-IPINV $r)(19)=r(46)$ and $($ DES-IPINV $r)(20)=r(14)$ and $(\mathrm{DES}-I P I N V r)(21)=r(54)$ and $($ DES-IPINV $r)(22)=r(22)$ and $($ DES-IPINV $r)(23)=r(62)$ and $($ DES-IPINV $r)(24)=r(30)$ and $($ DES-IPINV $r)(25)=r(37)$ and $($ DES-IPINV $r)(26)=r(5)$ and $($ DES-IPINV $r)(27)=r(45)$ and $($ DES-IPINV $r)(28)=r(13)$ and $(\mathrm{DES}-\mathrm{IPINV} r)(29)=r(53)$ and $(\mathrm{DES}-\mathrm{IPINV} r)(30)=r(21)$ and $($ DES-IPINV $r)(31)=r(61)$ and $($ DES-IPINV $r)(32)=r(29)$ and $($ DES-IPINV $r)(33)=r(36)$ and $($ DES-IPINV $r)(34)=r(4)$ and $($ DES-IPINV $r)(35)=r(44)$ and $($ DES-IPINV $r)(36)=r(12)$ and $(\mathrm{DES}-\mathrm{IPINV} r)(37)=r(52)$ and $(\mathrm{DES}-\mathrm{IPINV} r)(38)=r(20)$ and $($ DES-IPINV $r)(39)=r(60)$ and $($ DES-IPINV $r)(40)=r(28)$ and $($ DES-IPINV $r)(41)=r(35)$ and $($ DES-IPINV $r)(42)=r(3)$ and $($ DES-IPINV $r)(43)=r(43)$ and $($ DES-IPINV $r)(44)=r(11)$ and $(\mathrm{DES}-I P I N V r)(45)=r(51)$ and $($ DES-IPINV $r)(46)=r(19)$ and $($ DES-IPINV $r)(47)=r(59)$ and $($ DES-IPINV $r)(48)=r(27)$ and $($ DES-IPINV $r)(49)=r(34)$ and $($ DES-IPINV $r)(50)=r(2)$ and $($ DES-IPINV $r)(51)=r(42)$ and $($ DES-IPINV $r)(52)=r(10)$ and $(\mathrm{DES}-I P I N V r)(53)=r(50)$ and $(\mathrm{DES}-I P I N V r)(54)=r(18)$ and $(\mathrm{DES}-\mathrm{IPINV} r)(55)=r(58)$ and $(\mathrm{DES}-\mathrm{IPINV} r)(56)=r(26)$ and $($ DES-IPINV $r)(57)=r(33)$ and $($ DES-IPINV $r)(58)=r(1)$ and $($ DES-IPINV $r)(59)=r(41)$ and $($ DES-IPINV $r)(60)=r(9)$ and $(\mathrm{DES}-\mathrm{IPINV} r)(61)=r(49)$ and $(\mathrm{DES}-\mathrm{IPINV} r)(62)=r(17)$ and 
$($ DES-IPINV $r)(63)=r(57)$ and $($ DES-IPINV $r)(64)=r(25)$.

The function DES-PIPINV from Boolean ${ }^{64}$ into Boolean ${ }^{64}$ is defined by:

(Def. 17) For every element $i$ of Boolean ${ }^{64}$ holds $($ DES-PIPINV) $(i)=$ DES-IPINV $i$.

Let us note that DES-PIP is bijective.

Let us note that DES-PIPINV is bijective.

The following proposition is true

DES-PIPINV $=(\text { DES-PIP })^{-1}$.

\section{Feistel Function}

Let $r$ be an element of Boolean ${ }^{32}$. The functor DES-E $r$ yielding an element of Boolean ${ }^{48}$ is defined by the conditions (Def. 18).

(Def. 18) $\quad($ DES-E $r)(1)=r(32)$ and $($ DES-E $r)(2)=r(1)$ and $($ DES-E $r)(3)=r(2)$ and $(\mathrm{DES}-\mathrm{E} r)(4)=r(3)$ and $(\mathrm{DES}-\mathrm{E} r)(5)=r(4)$ and $(\mathrm{DES}-\mathrm{E} r)(6)=$ $r(5)$ and $(\mathrm{DES}-\mathrm{E} r)(7)=r(4)$ and $(\mathrm{DES}-\mathrm{E} r)(8)=r(5)$ and $(\mathrm{DES}-\mathrm{E} r)(9)=r(6)$ and $(\mathrm{DES}-\mathrm{E} r)(10)=r(7)$ and $(\mathrm{DES}-\mathrm{E} r)(11)=r(8)$ and $($ DES-E $r)(12)=r(9)$ and $($ DES-E $r)(13)=r(8)$ and $($ DES-E $r)(14)=$ $r(9)$ and $($ DES-E $r)(15)=r(10)$ and $($ DES-E $r)(16)=r(11)$ and $($ DES-E $r)(17)=r(12)$ and $($ DES-E $r)(18)=r(13)$ and $($ DES-E $r)(19)=$ $r(12)$ and $(\mathrm{DES}-\mathrm{E} r)(20)=r(13)$ and $(\mathrm{DES}-\mathrm{E} r)(21)=r(14)$ and $(\mathrm{DES}-\mathrm{E} r)(22)=r(15)$ and $(\mathrm{DES}-\mathrm{E} r)(23)=r(16)$ and (DES-E $r)(24)=$ $r(17)$ and $(\mathrm{DES}-\mathrm{E} r)(25)=r(16)$ and $(\mathrm{DES}-\mathrm{E} r)(26)=r(17)$ and $($ DES-E $r)(27)=r(18)$ and $($ DES-E $r)(28)=r(19)$ and $($ DES-E $r)(29)=$ $r(20)$ and $(\mathrm{DES}-\mathrm{E} r)(30)=r(21)$ and $(\mathrm{DES}-\mathrm{E} r)(31)=r(20)$ and $($ DES-E $r)(32)=r(21)$ and $($ DES-E $r)(33)=r(22)$ and $($ DES-E $r)(34)=$ $r(23)$ and $(\mathrm{DES}-\mathrm{E} r)(35)=r(24)$ and $(\mathrm{DES}-\mathrm{E} r)(36)=r(25)$ and $(\mathrm{DES}-\mathrm{E} r)(37)=r(24)$ and $(\mathrm{DES}-\mathrm{E} r)(38)=r(25)$ and $(\mathrm{DES}-\mathrm{E} r)(39)=$ $r(26)$ and $(\mathrm{DES}-\mathrm{E} r)(40)=r(27)$ and $(\mathrm{DES}-\mathrm{E} r)(41)=r(28)$ and $(\mathrm{DES}-\mathrm{E} r)(42)=r(29)$ and $(\mathrm{DES}-\mathrm{E} r)(43)=r(28)$ and $(\mathrm{DES}-\mathrm{E} r)(44)=$ $r(29)$ and $(\mathrm{DES}-\mathrm{E} r)(45)=r(30)$ and $(\mathrm{DES}-\mathrm{E} r)(46)=r(31)$ and $(\mathrm{DES}-\mathrm{E} r)(47)=r(32)$ and $(\mathrm{DES}-\mathrm{E} r)(48)=r(1)$.

Let $r$ be an element of Boolean ${ }^{32}$. The functor DES-P $r$ yielding an element of Boolean ${ }^{32}$ is defined by the conditions (Def. 19).

(Def. 19) $($ DES-P $r)(1)=r(16)$ and $($ DES-P $r)(2)=r(7)$ and $($ DES-P $r)(3)=$ $r(20)$ and $(\mathrm{DES}-\mathrm{P} r)(4)=r(21)$ and $(\mathrm{DES}-\mathrm{P} r)(5)=r(29)$ and $(\mathrm{DES}-\mathrm{P} r)(6)=r(12)$ and $(\mathrm{DES}-\mathrm{P} r)(7)=r(28)$ and $(\mathrm{DES}-\mathrm{P} r)(8)=$ $r(17)$ and $(\mathrm{DES}-\mathrm{P} r)(9)=r(1)$ and $(\mathrm{DES}-\mathrm{P} r)(10)=r(15)$ and $($ DES-P $r)(11)=r(23)$ and $($ DES-P $r)(12)=r(26)$ and $($ DES-P $r)(13)=$ $r(5)$ and $(\mathrm{DES}-\mathrm{P} r)(14)=r(18)$ and $(\mathrm{DES}-\mathrm{P} r)(15)=r(31)$ and 
$($ DES-P $r)(16)=r(10)$ and $($ DES-P $r)(17)=r(2)$ and $($ DES-P $r)(18)=$ $r(8)$ and $($ DES-P $r)(19)=r(24)$ and $($ DES-P $r)(20)=r(14)$ and $($ DES-P $r)(21)=r(32)$ and $($ DES-P $r)(22)=r(27)$ and $($ DES-P $r)(23)=$ $r(3)$ and $($ DES-P $r)(24)=r(9)$ and $($ DES-P $r)(25)=r(19)$ and $($ DES-P $r)(26)=r(13)$ and $($ DES-P $r)(27)=r(30)$ and $($ DES-P $r)(28)=$ $r(6)$ and $(\mathrm{DES}-\mathrm{P} r)(29)=r(22)$ and $(\mathrm{DES}-\mathrm{P} r)(30)=r(11)$ and $(\mathrm{DES}-\mathrm{P} r)(31)=r(4)$ and $(\mathrm{DES}-\mathrm{P} r)(32)=r(25)$.

Let $r$ be an element of Boolean ${ }^{48}$. The functor DES-DIV8 $r$ yielding an element of $\left(\text { Boolean }^{6}\right)^{8}$ is defined by the conditions (Def. 20).

$($ Def. 20$) \quad($ DES-DIV8 $r)(1)=$ Op-Left $(r, 6)$ and $($ DES-DIV8 $r)(2)=$ Op-Left $(\mathrm{Op}-\operatorname{Right}(r, 6), 6)$ and $(\mathrm{DES}-\mathrm{DIV} 8 r)(3)=$ Op-Left $($ Op-Right $(r, 12), 6)$ and $($ DES-DIV $8 r)(4)=$ Op-Left $(\mathrm{Op}-\operatorname{Right}(r, 18), 6)$ and $(\mathrm{DES}-\mathrm{DIV} 8 r)(5)=$ Op-Left $($ Op-Right $(r, 24), 6)$ and $($ DES-DIV8 $r)(6)=$ Op-Left $($ Op-Right $(r, 30), 6)$ and $($ DES-DIV $8 r)(7)=$ Op-Left $($ Op-Right $(r, 36), 6)$ and $(\operatorname{DES}-\mathrm{DIV} 8 r)(8)=$ Op-Right $(r, 42)$.

Next we state the proposition

(37) Let $r$ be an element of Boolean ${ }^{48}$. Then there exist elements $s_{1}, s_{2}$, $s_{3}, s_{4}, s_{5}, s_{6}, s_{7}, s_{8}$ of Boolean ${ }^{6}$ such that $s_{1}=($ DES-DIV $8 r)(1)$ and $s_{2}=($ DES-DIV8 $r)(2)$ and $s_{3}=($ DES-DIV $8 r)(3)$ and $s_{4}=$ $(\mathrm{DES}-\mathrm{DIV} 8 r)(4)$ and $s_{5}=(\mathrm{DES}-\mathrm{DIV} 8 r)(5)$ and $s_{6}=(\mathrm{DES}-\mathrm{DIV} 8 r)(6)$ and $s_{7}=($ DES-DIV $8 r)(7)$ and $s_{8}=($ DES-DIV8 $r)(8)$ and $r=s_{1} \frown s_{2} \frown$ $s_{3} \frown s_{4} \frown s_{5} \frown s_{6} \frown s_{7} \frown s_{8}$.

Let $t$ be an element of Boolean ${ }^{6}$. The functor B6toN64t yielding an element of 64 is defined by:

(Def. 21) B6toN64t $t=32 \cdot t(1)+16 \cdot t(6)+8 \cdot t(2)+4 \cdot t(3)+2 \cdot t(4)+1 \cdot t(5)$.

The function N16toB4 from 16 into Boolean ${ }^{4}$ is defined by the conditions (Def. 22).

(Def. 22) $(\mathrm{N} 16 \mathrm{toB} 4)(0)=\langle 0,0,0,0\rangle$ and $(\mathrm{N} 16 \mathrm{toB} 4)(1)=\langle 0,0,0,1\rangle$ and $(\mathrm{N} 16 \mathrm{toB} 4)(2)=\langle 0,0,1,0\rangle$ and $(\mathrm{N} 16 \mathrm{toB} 4)(3)=\langle 0,0,1,1\rangle$ and $(\mathrm{N} 16 \mathrm{toB} 4)(4)=\langle 0,1,0,0\rangle$ and $(\mathrm{N} 16 \mathrm{toB} 4)(5)=\langle 0,1,0,1\rangle$ and $(\mathrm{N} 16 \mathrm{toB} 4)(6)=\langle 0,1,1,0\rangle$ and $(\mathrm{N} 16 \mathrm{toB} 4)(7)=\langle 0,1,1,1\rangle$ and $(\mathrm{N} 16 \mathrm{toB} 4)(8)=\langle 1,0,0,0\rangle$ and $(\mathrm{N} 16 \mathrm{toB} 4)(9)=\langle 1,0,0,1\rangle$ and $(\mathrm{N} 16 \mathrm{toB} 4)(10)=\langle 1,0,1,0\rangle$ and $(\mathrm{N} 16 \mathrm{toB} 4)(11)=\langle 1,0,1,1\rangle$ and $(\mathrm{N} 16 \mathrm{toB} 4)(12)=\langle 1,1,0,0\rangle$ and $(\mathrm{N} 16 \mathrm{toB} 4)(13)=\langle 1,1,0,1\rangle$ and $(\mathrm{N} 16 \mathrm{toB} 4)(14)=\langle 1,1,1,0\rangle$ and $(\mathrm{N} 16 \mathrm{toB} 4)(15)=\langle 1,1,1,1\rangle$.

Let $R$ be an element of Boolean ${ }^{32}$ and let $R_{2}$ be an element of Boolean ${ }^{48}$. The functor DES-F $\left(R, R_{2}\right)$ yields an element of Boolean ${ }^{32}$ and is defined by the condition (Def. 23).

(Def. 23) There exist elements $D_{1}, D_{2}, D_{3}, D_{4}, D_{5}, D_{6}, D_{7}, D_{8}$ of Boolean ${ }^{6}$ and 
there exist elements $x_{1}, x_{2}, x_{3}, x_{4}, x_{5}, x_{6}, x_{7}, x_{8}$ of Boolean ${ }^{4}$ and there exists an element $C_{32}$ of Boolean ${ }^{32}$ such that

$D_{1}=\left(\right.$ DES-DIV8 Op-XOR $\left(\right.$ DES-E $\left.\left.R, R_{2}\right)\right)(1)$ and

$D_{2}=\left(\right.$ DES-DIV8 Op-XOR $\left(\right.$ DES-E $\left.\left.R, R_{2}\right)\right)(2)$ and

$D_{3}=\left(\mathrm{DES}-\mathrm{DIV} 8 \mathrm{Op}-\mathrm{XOR}\left(\mathrm{DES}-\mathrm{E} R, R_{2}\right)\right)(3)$ and

$D_{4}=\left(\mathrm{DES}-\mathrm{DIV} 8\right.$ Op-XOR $\left.\left(\mathrm{DES}-\mathrm{E} R, R_{2}\right)\right)(4)$ and

$D_{5}=\left(\mathrm{DES}-\mathrm{DIV} 8 \mathrm{Op}-\mathrm{XOR}\left(\mathrm{DES}-\mathrm{E} R, R_{2}\right)\right)(5)$ and

$D_{6}=\left(\mathrm{DES}-\mathrm{DIV} 8 \mathrm{Op}-\mathrm{XOR}\left(\mathrm{DES}-\mathrm{E} R, R_{2}\right)\right)(6)$ and

$D_{7}=\left(\right.$ DES-DIV8 Op-XOR $\left(\right.$ DES-E $\left.\left.R, R_{2}\right)\right)(7)$ and

$D_{8}=\left(\right.$ DES-DIV8 Op-XOR $\left(\right.$ DES-E $\left.\left.R, R_{2}\right)\right)(8)$ and

Op-XOR(DES-E $\left.R, R_{2}\right)=D_{1} \frown D_{2} \frown D_{3} \frown D_{4} \frown D_{5} \frown D_{6} \frown D_{7} \frown$ $D_{8}$ and $x_{1}=(\mathrm{N} 16 \mathrm{toB} 4)\left((\mathrm{DES}-\mathrm{SBOX} 1)\left(\mathrm{B} 6 \mathrm{toN} 64 D_{1}\right)\right)$ and $x_{2}=$ $(\mathrm{N} 16$ toB4 $)\left((\mathrm{DES}-\mathrm{SBOX} 2)\left(\mathrm{B} 6\right.\right.$ toN64 $\left.\left.D_{2}\right)\right)$ and

$x_{3}=(\mathrm{N} 16$ toB 4$)\left((\mathrm{DES}-\mathrm{SBOX} 3)\left(\mathrm{B} 6 \mathrm{toN} 64 D_{3}\right)\right)$ and

$x_{4}=(\mathrm{N} 16 \mathrm{toB} 4)\left((\mathrm{DES}-\mathrm{SBOX} 4)\left(\mathrm{B} 6 \mathrm{toN} 64 D_{4}\right)\right)$ and

$x_{5}=(\mathrm{N} 16 \mathrm{toB} 4)\left((\mathrm{DES}-\mathrm{SBOX} 5)\left(\mathrm{B} 6 \mathrm{toN} 64 D_{5}\right)\right)$ and

$x_{6}=(\mathrm{N} 16 \mathrm{toB} 4)\left((\mathrm{DES}-\mathrm{SBOX} 6)\left(\mathrm{B} 6 \mathrm{toN} 64 D_{6}\right)\right)$ and

$x_{7}=(\mathrm{N} 16 \mathrm{toB} 4)\left((\mathrm{DES}-\mathrm{SBOX} 7)\left(\mathrm{B} 6 \mathrm{toN} 64 D_{7}\right)\right)$ and

$x_{8}=(\mathrm{N} 16 \mathrm{toB} 4)\left((\mathrm{DES}-\mathrm{SBOX} 8)\left(\mathrm{B} 6 \mathrm{toN} 64 D_{8}\right)\right)$ and $C_{32}=x_{1} \frown x_{2} \frown x_{3} \frown$

$x_{4} \frown x_{5} \frown x_{6} \frown x_{7} \frown x_{8}$ and $\operatorname{DES}-\mathrm{F}\left(R, R_{2}\right)=\operatorname{DES}-\mathrm{P} C_{32}$.

The function DES-FFUNC from Boolean ${ }^{32} \times$ Boolean $^{48}$ into Boolean ${ }^{32}$ is defined as follows:

(Def. 24) For every element $z$ of Boolean ${ }^{32} \times$ Boolean $^{48}$ holds $($ DES-FFUNC) $(z)=$ $\operatorname{DES}-\mathrm{F}\left(z_{1}, z_{2}\right)$.

\section{Key Schedule}

Let $r$ be an element of Boolean ${ }^{64}$. The functor DES-PC1 $r$ yields an element of Boolean ${ }^{56}$ and is defined by the conditions (Def. 25).

$($ Def. 25$)($ DES-PC1 $r)(1)=r(57)$ and $($ DES-PC1 $r)(2)=r(49)$ and $(\mathrm{DES}-\mathrm{PC} 1 r)(3)=r(41)$ and $(\mathrm{DES}-\mathrm{PC} 1 r)(4)=r(33)$ and $(\mathrm{DES}-\mathrm{PC} 1 r)(5)=r(25)$ and $(\mathrm{DES}-\mathrm{PC} 1 r)(6)=r(17)$ and $(\mathrm{DES}-\mathrm{PC} 1 r)(7)=r(9)$ and $(\mathrm{DES}-\mathrm{PC} 1 r)(8)=r(1)$ and $(\mathrm{DES}-\mathrm{PC} 1 r)(9)=$ $r(58)$ and $(\mathrm{DES}-\mathrm{PC} 1 r)(10)=r(50)$ and $(\mathrm{DES}-\mathrm{PC} 1 r)(11)=r(42)$ and $(\mathrm{DES}-\mathrm{PC} 1 r)(12)=r(34)$ and $(\mathrm{DES}-\mathrm{PC} 1 r)(13)=r(26)$ and $(\mathrm{DES}-\mathrm{PC} 1 r)(14)=r(18)$ and $(\mathrm{DES}-\mathrm{PC} 1 r)(15)=r(10)$ and $(\mathrm{DES}-\mathrm{PC} 1 r)(16)=r(2)$ and $(\mathrm{DES}-\mathrm{PC} 1 r)(17)=r(59)$ and $(\mathrm{DES}-\mathrm{PC} 1 r)(18)=r(51)$ and $(\mathrm{DES}-\mathrm{PC} 1 r)(19)=r(43)$ and $(\mathrm{DES}-\mathrm{PC} 1 r)(20)=r(35)$ and $(\mathrm{DES}-\mathrm{PC} 1 r)(21)=r(27)$ and $(\mathrm{DES}-\mathrm{PC} 1 r)(22)=r(19)$ and $(\mathrm{DES}-\mathrm{PC} 1 r)(23)=r(11)$ and $(\mathrm{DES}-\mathrm{PC} 1 r)(24)=r(3)$ and $(\mathrm{DES}-\mathrm{PC} 1 r)(25)=r(60)$ and 


\begin{tabular}{|c|c|c|c|c|c|}
\hline$(\mathrm{DES}-\mathrm{PC} 1 r)(26)$ & $r(52)$ & and & $(\mathrm{DES}-\mathrm{PC} 1 r)(27)$ & $=$ & $r(44)$ \\
\hline$(\mathrm{DES}-\mathrm{PC} 1 r)(28)$ & $r(36)$ & and & $(\mathrm{DES}-\mathrm{PC} 1 r)(29)$ & $=$ & $r(63)$ \\
\hline$(\mathrm{DES}-\mathrm{PC} 1 r)(30)$ & $r(55)$ & and & $(\mathrm{DES}-\mathrm{PC} 1 r)(31)$ & $=$ & $r(47)$ \\
\hline$(\mathrm{DES}-\mathrm{PC} 1 r)(32)$ & $r(39)$ & and & $(\mathrm{DES}-\mathrm{PC} 1 r)(33)$ & $=$ & $r(31)$ \\
\hline$(\mathrm{DES}-\mathrm{PC} 1 r)(34)$ & $r(23)$ & and & $(\mathrm{DES}-\mathrm{PC} 1 r)(35)$ & $=$ & $r(15)$ \\
\hline$(\mathrm{DES}-\mathrm{PC} 1 r)(36)$ & $r(7)$ & and & $(\mathrm{DES}-\mathrm{PC} 1 r)(37)$ & $=$ & $r(62)$ \\
\hline$(\mathrm{DES}-\mathrm{PC} 1 r)(38)$ & $r(54)$ & and & $(\mathrm{DES}-\mathrm{PC} 1 r)(39)$ & $=$ & $r(46)$ \\
\hline$(\mathrm{DES}-\mathrm{PC} 1 r)(40)$ & $r(38)$ & and & $(\mathrm{DES}-\mathrm{PC} 1 r)(41)$ & $=$ & $r(30)$ \\
\hline$(\mathrm{DES}-\mathrm{PC} 1 r)(42)$ & $r(22)$ & and & $(\mathrm{DES}-\mathrm{PC} 1 r)(43)$ & $=$ & $r(14)$ \\
\hline$(\mathrm{DES}-\mathrm{PC} 1 r)(44)$ & $r(6)$ & and & $(\mathrm{DES}-\mathrm{PC} 1 r)(45)$ & $=$ & $r(61)$ \\
\hline$(\mathrm{DES}-\mathrm{PC} 1 r)(46)$ & $r(53)$ & and & $(\mathrm{DES}-\mathrm{PC} 1 r)(47)$ & $=$ & $r(45)$ \\
\hline$(\mathrm{DES}-\mathrm{PC} 1 r)(48)$ & $r(37)$ & and & $(\mathrm{DES}-\mathrm{PC} 1 r)(49)$ & $=$ & $r(29)$ \\
\hline$(\mathrm{DES}-\mathrm{PC} 1 r)(50)$ & $r(21)$ & and & $(\mathrm{DES}-\mathrm{PC} 1 r)(51)$ & $=$ & $r(13)$ \\
\hline$(\mathrm{DES}-\mathrm{PC} 1 r)(52)$ & $r(5)$ & and & $(\mathrm{DES}-\mathrm{PC} 1 r)(53)$ & $=$ & $r(28)$ \\
\hline$(\mathrm{DES}-\mathrm{PC} 1 r)(54)$ & $r(20)$ & and & $(\mathrm{DES}-\mathrm{PC} 1 r)(55)$ & $=$ & $r(12)$ \\
\hline
\end{tabular}

Let $r$ be an element of Boolean ${ }^{56}$. The functor DES-PC2 $r$ yielding an element of Boolean ${ }^{48}$ is defined by the conditions (Def. 26).

$($ Def. 26) $($ DES-PC2 $r)(1)=r(14)$ and $($ DES-PC2 $r)(2)=r(17)$ and $(\mathrm{DES}-\mathrm{PC} 2 r)(3)=r(11)$ and $(\mathrm{DES}-\mathrm{PC} 2 r)(4)=r(24)$ and $(\mathrm{DES}-\mathrm{PC} 2 r)(5)=r(1)$ and $(\mathrm{DES}-\mathrm{PC} 2 r)(6)=r(5)$ and $(\mathrm{DES}-\mathrm{PC} 2 r)(7)=$ $r(3)$ and $(\mathrm{DES}-\mathrm{PC} 2 r)(8)=r(28)$ and $(\mathrm{DES}-\mathrm{PC} 2 r)(9)=r(15)$ and $(\mathrm{DES}-\mathrm{PC} 2 r)(10)=r(6)$ and $(\mathrm{DES}-\mathrm{PC} 2 r)(11)=r(21)$ and $(\mathrm{DES}-\mathrm{PC} 2 r)(12)=r(10)$ and $(\mathrm{DES}-\mathrm{PC} 2 r)(13)=r(23)$ and $(\mathrm{DES}-\mathrm{PC} 2 r)(14)=r(19)$ and $(\mathrm{DES}-\mathrm{PC} 2 r)(15)=r(12)$ and $(\mathrm{DES}-\mathrm{PC} 2 r)(16)=r(4)$ and $(\mathrm{DES}-\mathrm{PC} 2 r)(17)=r(26)$ and $(\mathrm{DES}-\mathrm{PC} 2 r)(18)=r(8)$ and $(\mathrm{DES}-\mathrm{PC} 2 r)(19)=r(16)$ and $(\mathrm{DES}-\mathrm{PC} 2 r)(20)=r(7)$ and $(\mathrm{DES}-\mathrm{PC} 2 r)(21)=r(27)$ and $(\mathrm{DES}-\mathrm{PC} 2 r)(22)=r(20)$ and $(\mathrm{DES}-\mathrm{PC} 2 r)(23)=r(13)$ and $(\mathrm{DES}-\mathrm{PC} 2 r)(24)=r(2)$ and $(\mathrm{DES}-\mathrm{PC} 2 r)(25)=r(41)$ and $(\mathrm{DES}-\mathrm{PC} 2 r)(26)=r(52)$ and $(\mathrm{DES}-\mathrm{PC} 2 r)(27)=r(31)$ and $(\mathrm{DES}-\mathrm{PC} 2 r)(28)=r(37)$ and $(\mathrm{DES}-\mathrm{PC} 2 r)(29)=r(47)$ and $(\mathrm{DES}-\mathrm{PC} 2 r)(30)=r(55)$ and $(\mathrm{DES}-\mathrm{PC} 2 r)(31)=r(30)$ and $(\mathrm{DES}-\mathrm{PC} 2 r)(32)=r(40)$ and $(\mathrm{DES}-\mathrm{PC} 2 r)(33)=r(51)$ and $(\mathrm{DES}-\mathrm{PC} 2 r)(34)=r(45)$ and $(\mathrm{DES}-\mathrm{PC} 2 r)(35)=r(33)$ and $(\mathrm{DES}-\mathrm{PC} 2 r)(36)=r(48)$ and $(\mathrm{DES}-\mathrm{PC} 2 r)(37)=r(44)$ and $(\mathrm{DES}-\mathrm{PC} 2 r)(38)=r(49)$ and $(\mathrm{DES}-\mathrm{PC} 2 r)(39)=r(39)$ and $(\mathrm{DES}-\mathrm{PC} 2 r)(40)=r(56)$ and $(\mathrm{DES}-\mathrm{PC} 2 r)(41)=r(34)$ and $(\mathrm{DES}-\mathrm{PC} 2 r)(42)=r(53)$ and $(\mathrm{DES}-\mathrm{PC} 2 r)(43)=r(46)$ and $(\mathrm{DES}-\mathrm{PC} 2 r)(44)=r(42)$ and $(\mathrm{DES}-\mathrm{PC} 2 r)(45)=r(50)$ and $(\mathrm{DES}-\mathrm{PC} 2 r)(46)=r(36)$ and $(\mathrm{DES}-\mathrm{PC} 2 r)(47)=r(29)$ and 
$($ DES-PC2 $r)(48)=r(32)$.

The finite sequence bitshift $t_{\mathrm{DES}}$ of elements of $\mathbb{N}$ is defined by the conditions (Def. 27).

(Def. 27) bitshift $_{\mathrm{DES}}$ is 16-element and $\left(\right.$ bitshift $\left._{\mathrm{DES}}\right)(1)=1$ and $\left(\right.$ bitshift $\left._{\mathrm{DES}}\right)(2)=$ 1 and $\left(\operatorname{bitshift}_{\mathrm{DES}}\right)(3)=2$ and $\left(\operatorname{bitshift}_{\mathrm{DES}}\right)(4)=2$ and $\left(\right.$ bitshift $\left._{\mathrm{DES}}\right)(5)=$ 2 and $\left(\right.$ bitshift $\left._{\mathrm{DES}}\right)(6)=2$ and $\left(\right.$ bitshift $\left._{\mathrm{DES}}\right)(7)=2$ and $\left(\right.$ bitshift $\left._{\mathrm{DES}}\right)(8)=$ 2 and $\left(\right.$ bitshift $\left._{\mathrm{DES}}\right)(9)=1$ and $\left(\right.$ bitshift $\left._{\mathrm{DES}}\right)(10)=2$ and $\left(\right.$ bitshift $\left._{\mathrm{DES}}\right)(11)=2$ and $\left(\right.$ bitshift $\left._{\mathrm{DES}}\right)(12)=2$ and $\left(\right.$ bitshift $\left._{\mathrm{DES}}\right)(13)=$ 2 and $\left(\right.$ bitshift $\left._{\mathrm{DES}}\right)(14)=2$ and $\left(\right.$ bitshift $\left._{\mathrm{DES}}\right)(15)=2$ and $\left(\right.$ bitshift $\left._{\mathrm{DES}}\right)(16)=1$.

Let $K_{1}$ be an element of Boolean ${ }^{64}$. The functor DES-KS $K_{1}$ yielding an element of $\left(\text { Boolean }^{48}\right)^{16}$ is defined by the condition (Def. 28).

(Def. 28) There exist sequences $C, D$ of Boolean ${ }^{28}$ such that

(i) $C(0)=$ Op-Left $\left(\right.$ DES-PC1 $\left.K_{1}, 28\right)$,

(ii) $\quad D(0)=$ Op-Right $\left(\right.$ DES-PC1 $\left.K_{1}, 28\right)$, and

(iii) for every element $i$ of $\mathbb{N}$ such that $0 \leq i \leq 15$ holds $\left(\right.$ DES-KS $\left.K_{1}\right)(i+1)=\operatorname{DES}-\mathrm{PC} 2(C(i+1) \frown D(i+1))$ and $C(i+1)=$ Op-Shift $\left(C(i),\left(\operatorname{bitshift}_{\mathrm{DES}}\right)(i)\right)$ and $D(i+1)=$ Op-Shift $\left(D(i)\right.$, bitshift $\left.\left._{\text {DES }}\right)(i)\right)$.

\section{ENCRYPTION AND DECRYPTION}

Let $n, m, k$ be non empty elements of $\mathbb{N}$, let $R_{1}$ be an element of $\left(\text { Boolean }^{m}\right)^{k}$, let $F$ be a function from Boolean ${ }^{n} \times$ Boolean $^{m}$ into Boolean ${ }^{n}$, let $I_{1}$ be a permutation of Boolean ${ }^{2 \cdot n}$, and let $M$ be an element of Boolean ${ }^{2 \cdot n}$. The functor DES-like-CoDec $\left(M, F, I_{1}, R_{1}\right)$ yields an element of Boolean ${ }^{2 \cdot n}$ and is defined by the condition (Def. 29).

(Def. 29) There exist sequences $L, R$ of Boolean ${ }^{n}$ such that

(i) $\quad L(0)=$ SP-Left $I_{1}(M)$

(ii) $\quad R(0)=$ SP-Right $I_{1}(M)$,

(iii) for every element $i$ of $\mathbb{N}$ such that $0 \leq i \leq k-1$ holds $L(i+1)=R(i)$ and $R(i+1)=\mathrm{Op}-\mathrm{XOR}\left(L(i), F\left(R(i),\left(R_{1}\right)_{i+1}\right)\right)$, and

(iv) $\operatorname{DES}-$ like-CoDec$\left(M, F, I_{1}, R_{1}\right)=I_{1}^{-1}(R(k) \frown L(k))$.

The following proposition is true

(38) Let $n, m, k$ be non empty elements of $\mathbb{N}, R_{1}$ be an element of $\left(\text { Boolean }^{m}\right)^{k}, F$ be a function from Boolean ${ }^{n} \times$ Boolean $^{m}$ into Boolean ${ }^{n}, I_{1}$ be a permutation of Boolean ${ }^{2 \cdot n}$, and $M$ be an element of Boolean ${ }^{2 \cdot n}$. Then DES-like-CoDec(DES-like-CoDec $\left.\left(M, F, I_{1}, R_{1}\right), F, I_{1}, \operatorname{Rev}\left(R_{1}\right)\right)=M$.

Let $R_{1}$ be an element of $\left(\text { Boolean }^{48}\right)^{16}$, let $F$ be a function from Boolean ${ }^{32} \times$ Boolean $^{48}$ into Boolean ${ }^{32}$, let $I_{1}$ be a permutation of Boolean ${ }^{64}$, and let $M$ be an 
element of Boolean ${ }^{64}$. The functor DES-CoDec $\left(M, F, I_{1}, R_{1}\right)$ yielding an element of Boolean ${ }^{64}$ is defined by:

(Def. 30) There exists a permutation $I_{2}$ of Boolean ${ }^{2 \cdot 32}$ and there exists an element $M_{1}$ of Boolean ${ }^{2 \cdot 32}$ such that $I_{2}=I_{1}$ and $M_{1}=M$ and $\operatorname{DES}-\operatorname{CoDec}\left(M, F, I_{1}, R_{1}\right)=\operatorname{DES}-$ like-CoDec $\left(M_{1}, F, I_{2}, R_{1}\right)$.

The following proposition is true

(39) Let $R_{1}$ be an element of $\left(\text { Boolean }^{48}\right)^{16}, F$ be a function from Boolean ${ }^{32} \times$ Boolean $^{48}$ into Boolean ${ }^{32}, I_{1}$ be a permutation of Boolean ${ }^{64}$, and $M$ be an element of Boolean ${ }^{64}$.

Then DES-CoDec(DES-CoDec $\left.\left(M, F, I_{1}, R_{1}\right), F, I_{1}, \operatorname{Rev}\left(R_{1}\right)\right)=M$.

Let $p_{1}, s_{9}$ be elements of Boolean ${ }^{64}$. The functor $\operatorname{DES-ENC}\left(p_{1}, s_{9}\right)$ yields an element of Boolean ${ }^{64}$ and is defined by:

(Def. 31$)$ DES-ENC $\left(p_{1}, s_{9}\right)=$ DES-CoDec $\left(p_{1}\right.$, DES-FFUNC, DES-PIP, DES-KS $\left.s_{9}\right)$.

Let $c_{1}, s_{9}$ be elements of Boolean ${ }^{64}$. The functor $\operatorname{DES}-\operatorname{DEC}\left(c_{1}, s_{9}\right)$ yields an element of Boolean ${ }^{64}$ and is defined as follows:

(Def. 32) DES-DEC $\left(c_{1}, s_{9}\right)=$

DES-CoDec $\left(c_{1}\right.$, DES-FFUNC, DES-PIP, Rev(DES-KS $\left.\left.s_{9}\right)\right)$.

The following proposition is true

(40) For all elements $m_{1}, s_{9}$ of Boolean ${ }^{64}$ holds

$\operatorname{DES}-\mathrm{DEC}\left(\operatorname{DES}-\operatorname{ENC}\left(m_{1}, s_{9}\right), s_{9}\right)=m_{1}$.

\section{REFERENCES}

[1] Grzegorz Bancerek. Cardinal numbers. Formalized Mathematics, 1(2):377-382, 1990.

[2] Grzegorz Bancerek. The fundamental properties of natural numbers. Formalized Mathematics, 1(1):41-46, 1990.

[3] Grzegorz Bancerek. The ordinal numbers. Formalized Mathematics, 1(1):91-96, 1990.

[4] Grzegorz Bancerek and Krzysztof Hryniewiecki. Segments of natural numbers and finite sequences. Formalized Mathematics, 1(1):107-114, 1990.

[5] Czesław Byliński. Binary operations. Formalized Mathematics, 1(1):175-180, 1990.

[6] Czesław Byliński. Finite sequences and tuples of elements of a non-empty sets. Formalized Mathematics, 1(3):529-536, 1990.

[7] Czesław Byliński. Functions and their basic properties. Formalized Mathematics, 1(1):55$65,1990$.

[8] Czesław Byliński. Functions from a set to a set. Formalized Mathematics, 1(1):153-164, 1990.

[9] Czesław Byliński. Partial functions. Formalized Mathematics, 1(2):357-367, 1990.

[10] Czesław Byliński. Some basic properties of sets. Formalized Mathematics, 1(1):47-53, 1990.

[11] Czesław Byliński. Some properties of restrictions of finite sequences. Formalized Mathematics, 5(2):241-245, 1996.

[12] Shunichi Kobayashi and Kui Jia. A theory of Boolean valued functions and partitions. Formalized Mathematics, 7(2):249-254, 1998.

[13] Jarosław Kotowicz. Functions and finite sequences of real numbers. Formalized Mathematics, 3(2):275-278, 1992.

[14] Takaya Nishiyama and Yasuho Mizuhara. Binary arithmetics. Formalized Mathematics, 4(1):83-86, 1993 
[15] U.S. Department of Commerce/National Institute of Standards and Technology. Fips pub 46-3, data encryption standard (DES). http://csrc.nist.gov/publications/fips/fips46-3/fips46-3.pdf. Federal Information Processing Standars Publication, 1999.

[16] Andrzej Trybulec. Domains and their Cartesian products. Formalized Mathematics, 1(1):115-122, 1990.

[17] Michał J. Trybulec. Integers. Formalized Mathematics, 1(3):501-505, 1990.

[18] Wojciech A. Trybulec. Pigeon hole principle. Formalized Mathematics, 1(3):575-579, 1990.

[19] Zinaida Trybulec. Properties of subsets. Formalized Mathematics, 1(1):67-71, 1990.

[20] Edmund Woronowicz. Many argument relations. Formalized Mathematics, 1(4):733-737, 1990.

[21] Edmund Woronowicz. Relations and their basic properties. Formalized Mathematics, 1(1):73-83, 1990.

[22] Edmund Woronowicz. Relations defined on sets. Formalized Mathematics, 1(1):181-186, 1990.

Received November 30, 2011 